\title{
A POPULATION OF X-RAY WEAK QUASARS: PHL 1811 ANALOGS AT HIGH REDSHIFT
}

\author{
Jianfeng Wu ${ }^{1,2}$, W. N. BrandT ${ }^{1,2}$, Patrick B. Hall ${ }^{3}$, Robert R. Gibson ${ }^{4}$, Gordon T. Richards ${ }^{5}$, \\ Donald P. Schneider ${ }^{1}$, Ohad Shemmer ${ }^{6}$, Dennis W. Just ${ }^{7}$, and Sarah J. Schmidt ${ }^{4}$ \\ ${ }^{1}$ Department of Astronomy \& Astrophysics, The Pennsylvania State University, 525 Davey Lab, University Park, PA 16802, USA; jfwu@astro.psu.edu \\ ${ }^{2}$ Institute for Gravitation and the Cosmos, The Pennsylvania State University, University Park, PA 16802, USA \\ ${ }^{3}$ Department of Physics \& Astronomy, York University, 4700 Keele Street, Toronto, ON M3J 1P3, Canada \\ ${ }^{4}$ Department of Astronomy, University of Washington, Box 351580, Seattle, WA 98195, USA \\ ${ }^{5}$ Department of Physics, Drexel University, 3141 Chestnut Street, Philadelphia, PA 19104, USA \\ ${ }^{6}$ Department of Physics, University of North Texas, Denton, TX 76203, USA \\ ${ }^{7}$ Steward Observatory, University of Arizona, 933 North Cherry Avenue, Tucson, AZ 85721, USA \\ Received 2011 January 17; accepted 2011 April 15; published 2011 July 1
}

\begin{abstract}
We report the results from Chandra and XMM-Newton observations of a sample of 10 type 1 quasars selected to have unusual UV emission-line properties (weak and blueshifted high-ionization lines; strong UV Fe emission) similar to those of PHL 1811, a confirmed intrinsically X-ray weak quasar. These quasars were identified by the Sloan Digital Sky Survey at high redshift $(z \approx 2.2)$; eight are radio quiet while two are radio intermediate. All of the radio-quiet PHL 1811 analogs, without exception, are notably X-ray weak by a mean factor of $\approx 13$. These sources lack broad absorption lines and have blue UV/optical continua, supporting the hypothesis that they are intrinsically X-ray weak like PHL 1811 itself. However, their average X-ray spectrum appears to be harder than those of typical quasars, which may indicate the presence of heavy intrinsic X-ray absorption. Our sample of radio-quiet PHL 1811 analogs supports a connection between an X-ray weak spectral energy distribution and PHL 1811-like UV emission lines; this connection provides an economical way to identify X-ray weak type 1 quasars. The fraction of radio-quiet PHL 1811 analogs in the radio-quiet quasar population is estimated to be $\lesssim 1.2 \%$. We have investigated correlations between relative X-ray brightness and UV emission-line properties (e.g., C IV equivalent width and blueshift) for a sample combining our radio-quiet PHL 1811 analogs, PHL 1811 itself, and typical type 1 quasars. These correlation analyses suggest that PHL 1811 analogs may have extreme wind-dominated broad emission-line regions. Observationally, the radio-quiet PHL 1811 analogs appear to be a subset $(\approx 30 \%)$ of radio-quiet weak-line quasars (WLQs). The existence of a subset of quasars in which high-ionization "shielding gas" covers most of the broad emission-line region (BELR), but little more than the BELR, could potentially unify the PHL 1811 analogs and WLQs. The two radio-intermediate PHL 1811 analogs are X-ray bright. X-ray spectral analyses and consideration of their multiwavelength properties suggest that one of them has jet-dominated X-ray emission, while the nature of the other remains unclear.
\end{abstract}

Key words: galaxies: active - galaxies: nuclei - quasars: emission lines - X-rays: galaxies

Online-only material: color figures

\section{INTRODUCTION}

A central tenet of X-ray astronomy is that luminous X-ray emission is a universal property of efficiently accreting supermassive black holes (SMBHs). This idea underlies the utility of extragalactic X-ray surveys for finding active galactic nuclei (AGNs) throughout the universe. While this tenet has generally withstood observational tests (e.g., Avni \& Tananbaum 1986; Mushotzky 2004; Brandt \& Hasinger 2005; Gibson et al. 2008a; Brandt \& Alexander 2010, and references therein), it is poorly understood physically. The accretion-disk corona (ADC), putatively responsible for creating most of the observed AGN X-ray emission via Compton upscattering of lower energy disk photons, is still not well understood, and its strong level of $\mathrm{X}$-ray emission cannot yet be reliably derived from ab initio physics (e.g., Galeev et al. 1979; Fabian et al. 2000; Miller \& Stone 2000; Krolik 2007).

Notably, there are a few examples of AGNs where the ADC appears to emit X-rays much more weakly, by a factor of $\approx 10-100$, than expected based upon the emission at longer wavelengths. These strange objects, which persist in showing spectral energy distributions (SEDs) entirely out of keeping with their luminosity, may ultimately teach us more than a host that radiate according to rule (cf. Eddington 1922). The best studied such case is the radio-quiet quasar PHL $1811(z=0.19$; Leighly et al. 2007a, 2007b). In multiple X-ray observations, this quasar has been found to be consistently X-ray weak relative to expectations from the $\alpha_{\mathrm{ox}}-L_{2500 \AA}$ relation by a factor of $\approx 30-100 .{ }^{8}$ Its X-ray spectrum shows no evidence for absorption of an intrinsically strong underlying X-ray continuum, and large-amplitude variability demonstrates that the X-rays are not strongly scattered in the nuclear region. PHL 1811 thus appears to be intrinsically X-ray weak. The ultimate physical reason for this intrinsic X-ray weakness is poorly understood. It may be due to a high accretion rate (i.e., $L / L_{\mathrm{Edd}}$ ) onto the $\mathrm{SMBH}$; this property could quench or catastrophically cool the ADC, perhaps due to "trapping" effects (e.g., Begelman 1978).

The UV/optical spectrum of PHL 1811 is also unusual (Leighly et al. 2007a). It shows no clear forbidden or semiforbidden line emission. The C IV $\lambda 1549$ emission line is weak by a factor of $\approx 5$ compared to composite quasar spectra (only

\footnotetext{
$8 \alpha_{\text {ox }}$ is defined to be the slope of a power law connecting the rest-frame $2500 \AA$ and $2 \mathrm{keV}$ monochromatic luminosities; i.e., $\alpha_{\mathrm{ox}}=0.3838$

$\log \left(L_{2 \mathrm{keV}} / L_{2500 \AA}\right)$. This quantity is well known to be correlated with $L_{2500 \AA}$ (e.g., Steffen et al. 2006 and references therein).
} 
$\approx 1 \%$ of SDSS quasars with similar luminosity have such weak C IV lines; e.g., Shen et al. 2011). The C IV line is also blueshifted and asymmetric; the observed C IV characteristics may indicate the presence of a strong radiatively driven wind that dominates the broad emission-line region (BELR; e.g., Richards et al. 2011). The near-UV spectrum is dominated by strong Fe II and Fe III emission lines, and unusual low-ionization emission lines such as $\mathrm{Na}$ I D and $\mathrm{Ca}$ II $\mathrm{H}$ and $\mathrm{K}$ are observed. Based on photoionization modeling, Leighly et al. (2007a) suggest that the unusual UV/optical emission-line properties of PHL 1811 are due to a weak ionizing continuum, inferred from its soft (i.e., X-ray weak and UV/optical strong) SED. This apparent connection between the SED and emission-line properties does not, unfortunately, directly clarify the ultimate physical cause of the intrinsic X-ray weakness. However, if correct, this suggestion should provide an economical method of identifying additional examples of intrinsically X-ray weak quasars from large optical spectroscopic databases. Investigations of these additional examples might then provide a broader context and insight into the cause of the intrinsic X-ray weakness. Additional evidence for a connection between a soft SED and PHL 1811-like emission-line properties comes from the finding that the extremely luminous quasar SDSS J1521+5202 $(z=$ $2.19 ; M_{i}=-30.19$ ) is also remarkably X-ray weak (by a factor of $\approx 30$; Just et al. 2007) and has similar UV emission-line properties to PHL 1811. However, the sample size remains too small to claim an empirical connection reliably.

One other recent result that is relevant to the issue of intrinsically X-ray weak quasars is the finding by Miniutti et al. (2009) of an abrupt and remarkable drop by a factor of $\approx 200$ in the X-ray luminosity of the well-studied quasar PHL 1092 $(z=0.40)$. This quasar appears to have made a strong transition between X-ray bright and X-ray weak states, perhaps associated with evolving instabilities in its ADC (see Miniutti et al. 2009; Sobolewska et al. 2009). Interestingly, PHL 1092 was noted to have similar emission-line properties to PHL 1811 prior to the discovery of its X-ray luminosity drop (Leighly et al. 2007a). Somewhat surprisingly, the wavelength region from $\mathrm{Mg}$ II to $\mathrm{H} \beta$ in PHL 1092 did not show dramatic changes associated with its transition from a normal SED to an X-ray weak SED (Miniutti et al. 2009).

Given the potential physical importance of quasars with PHL 1811-like emission-line properties (hereafter PHL 1811 analogs), we proposed to use Chandra to substantially enlarge the sample of these objects with sensitive X-ray coverage. Our observation targets were selected from the vast Sloan Digital Sky Survey (SDSS; York et al. 2000) spectroscopic database to be sufficiently optically bright to allow derivation of tight optical-to-X-ray SED constraints with short (5-13 ks) Chandra observations. We also searched for and utilized sensitive archival X-ray coverage of a few PHL 1811 analogs. The closely related goals of our project were the following: (1) to determine if there is evidence for a reasonably sized population of intrinsically $\mathrm{X}$-ray weak quasars. If such a population indeed exists, it would present an interesting challenge to the universality of luminous X-ray emission from quasars, and one that might lead to insights into when luminous ADCs do and do not form, (2) to assess empirically if there is a physical connection between soft (i.e., X-ray weak and UV/optical strong) quasar SEDs and PHL 1811-like emission-line properties, as has been proposed to be the case from photoionization modeling, (3) to investigate if selection upon PHL 1811-like emission-line properties provides a practical and economical way of finding X-ray weak quasars

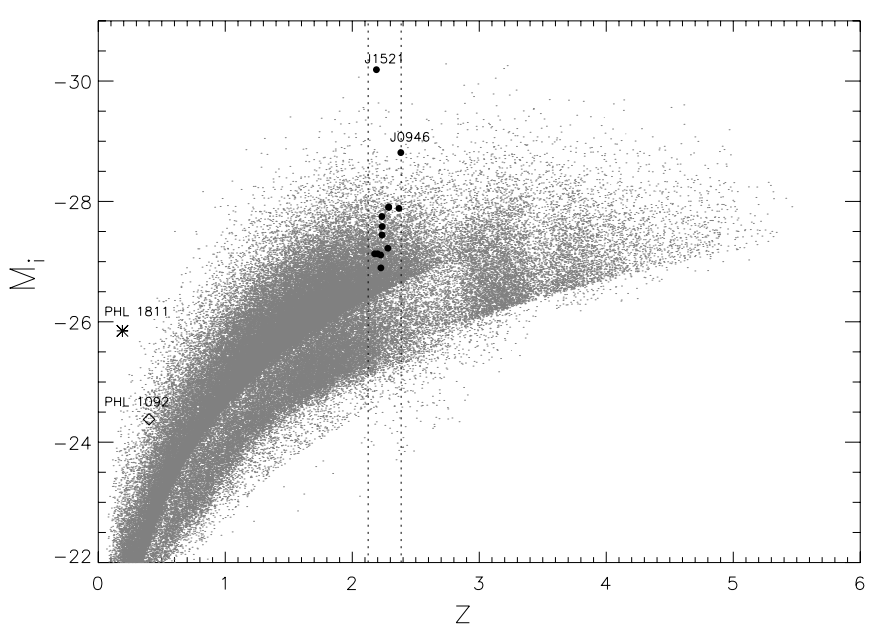

Figure 1. SDSS absolute $i$-band magnitude, $M_{i}$, plotted vs. redshift, $z$. The filled black circles show our sample of 11 selected quasars, the asterisk represents PHL 1811, the open diamond represents PHL 1092, and the small gray dots represent the 105,783 objects in the SDSS DR7 quasar catalog (Schneider et al. 2010). The two most luminous sources in our sample are labeled in the format of "Jhhmm" for brevity. The vertical dotted lines show the redshift criterion for our sample selection $(2.125 \leqslant z \leqslant 2.385)$.

at high redshift, and (4) to provoke further X-ray studies of PHL 1811 analogs. For example, X-ray spectroscopy of any Xray weak members of this class should be able to test directly if they, like PHL 1811, are intrinsically X-ray weak rather than absorbed, and it should also provide estimates of their $L / L_{\text {Edd }}$ values. Consistent $\mathrm{X}$-ray monitoring over long timescales might also reveal strong X-ray variability (perhaps associated with ADC instabilities), as has been found for PHL 1092.

In Section 2, we describe the selection of our sample of PHL 1811 analogs and measurements of their rest-frame UV spectral properties. In Section 3, we describe the relevant X-ray data analyses. Overall results and associated discussion are presented in Section 4. Throughout this paper, we adopt a cosmology with $H_{0}=70.5 \mathrm{~km} \mathrm{~s}^{-1} \mathrm{Mpc}^{-1}, \Omega_{\mathrm{M}}=0.274$, and $\Omega_{\Lambda}=0.726$ (e.g., Komatsu et al. 2009).

\section{SAMPLE SELECTION AND REST-FRAME UV SPECTRAL MEASUREMENTS}

\subsection{Selection of PHL 1811 Analogs}

We first compiled a sample of the 1621 objects classified as QSO or HIZ_QSO in the SDSS Data Release 7 (DR7; Abazajian et al. 2009) Catalog Archive Server (CAS) with $m_{r} \leqslant 18.8$ and $2.125 \leqslant z \leqslant 2.385$ (see Figure 1). The magnitude limit selects objects that are sufficiently bright for short Chandra observations to provide tight constraints on their optical-to-X-ray SED properties. The selected redshift range provides the best possible coverage between Ly $\alpha$ and Fe II (and usually $\mathrm{Mg}$ II) in the SDSS spectra, which allows reliable identification of PHL 1811 analogs. These analogs were systematically selected as quasars having weak and blueshifted high-ionization lines, such as C IV $\lambda 1549$ and Si IV $\lambda 1400,{ }^{9}$ as well as strong UV Fe II

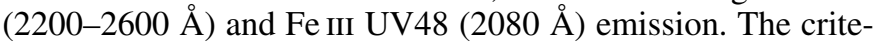
ria upon rest-frame equivalent widths $\left(W_{r}\right)$ of high-ionization lines extracted from the SDSS CAS SpecObj table were $W_{r}$ $(\mathrm{Si}$ IV $) \leqslant 15 \AA,-15 \AA \leqslant W_{r}(\mathrm{C}$ IV $) \leqslant 20 \AA$, and $\chi^{2} \leqslant 20$ for the $C$ IV line fit; the $\chi^{2}$ fit-quality criterion ensures reliable

\footnotetext{
9 This line is, in fact, a blend of Si IV and O IV]; we refer to it simply as Si IV for convenience.
} 

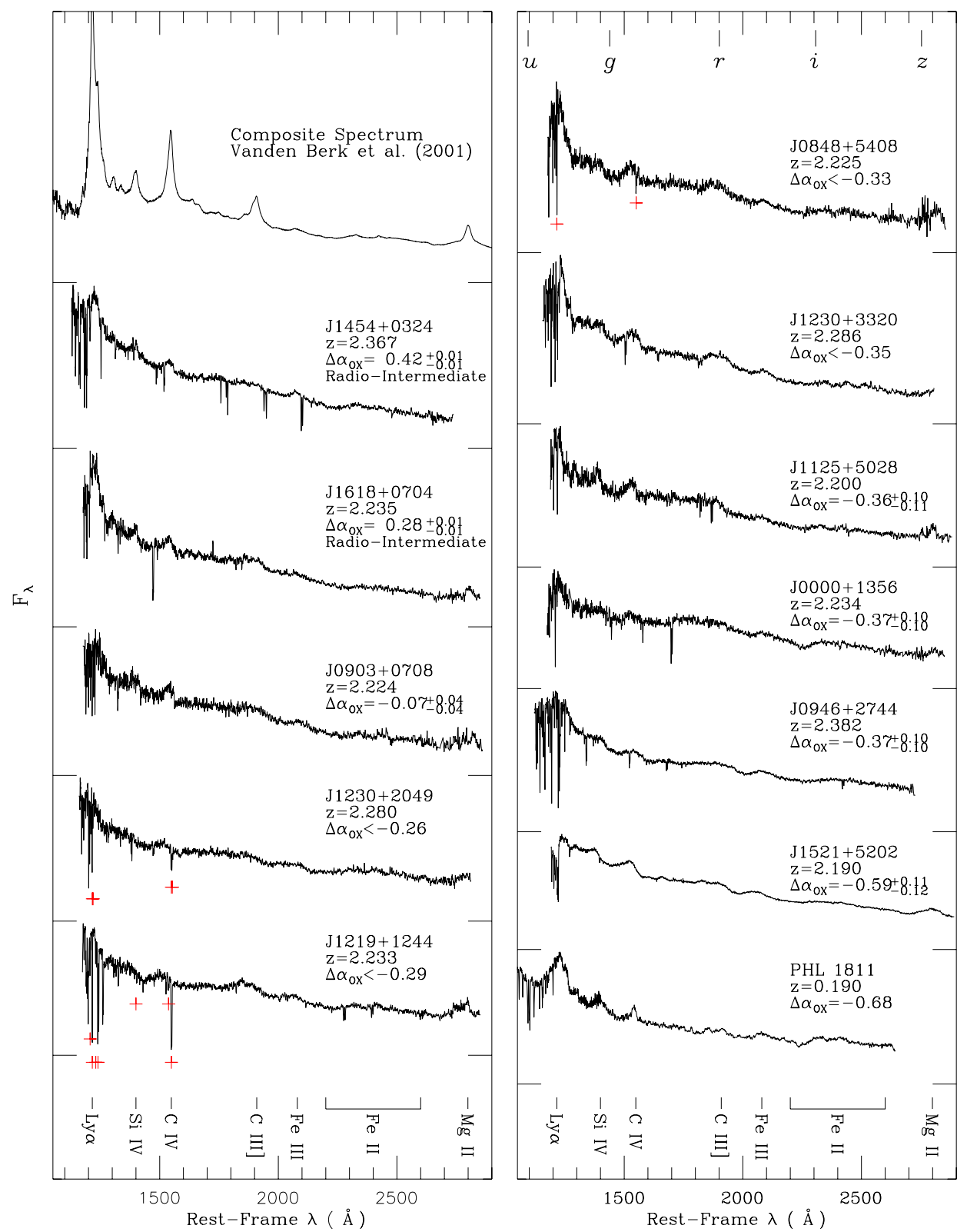

Figure 2. SDSS spectra for the 11 sources in our sample of potential PHL 1811 analogs (J0903+0708 is later excluded from our sample, see Section 2.3), ordered by $\Delta \alpha_{\text {ox }}$ (see Section 3 for definition). The $\Delta \alpha_{\text {ox }}$ values and their error bars (if the source is detected in X-rays) are shown for each source. The name of each source is labeled in the format of "Jhhmm+ddmm." The $y$-coordinates are the flux density $\left(F_{\lambda}\right)$ in arbitrary linear units. The tick marks on the $y$-axis show the zero flux density level for each normalized spectrum. The spectra have been smoothed using a five-pixel sliding-box filter. Emission lines, including Ly $\alpha \lambda 1216$, Si IV $\lambda 1400$, C IV $\lambda 1549$, Fe III $\lambda 2080$, Fe II $\lambda 2200-2600$, and Mg II $\lambda 2799$ are labeled in each panel. The associated narrow absorption lines are labeled with red " + " signs (the two associated NAL systems of J1230+2049 overlap each other because of their very close redshifts; see Table 2). The rest-frame effective wavelengths of the SDSS ugriz bands for the median redshift of our PHL 1811 analogs $(z=2.23)$ are labeled at the top of the right panel. The spectral resolution is $R \approx 2000$. Also included are the composite spectrum of SDSS quasars by Vanden Berk et al. (2001) and the PHL 1811 spectrum from HST (Leighly et al. 2007a).

(A color version of this figure is available in the online journal.)

measurements of C IV line properties. ${ }^{10}$ All 54 spectra meeting these criteria were then inspected visually by P.B.H. We first excluded sources that are misclassified stars or are otherwise spurious, as well as sources that have bad redshift measurements or detectable UV broad absorption lines (BALs). BAL quasars were excluded because they typically show substantial X-ray absorption (e.g., Gallagher et al. 2002, 2006; Gibson et al. 2009b). After this examination we had 32 sources that are non-BAL weak-line quasars (WLQs; see Section 4.6 for fur-

\footnotetext{
${ }^{10}$ We also required $W_{r}(\mathrm{Ly} \alpha) \leqslant 105 \AA$ and $W_{r}(\mathrm{~N}$ v $\lambda 1240) \leqslant 85 \AA$ to
} eliminate quasars with strong $\operatorname{Ly} \alpha+\mathrm{N} v$ blends. ther details). Among these 32 sources, objects with strong UV Fe II and Fe III UV48 emission and whose high-ionization lines showed a strong blueshifted component (with the magnitude of the blueshift exceeding $800 \mathrm{~km} \mathrm{~s}^{-1}$ ) were kept in the sample. We rejected 13 objects primarily due to weak $\mathrm{Fe}$ emission, and 8 objects primarily due to a low or unknown blueshift. After doing this, 11 quasars remained in our sample. ${ }^{11}$ Figure 2 shows the SDSS spectra for these potential PHL 1811 analogs. The SDSS quasar composite spectrum from Vanden Berk et al. (2001) and

\footnotetext{
11 One radio-quiet source, J0903+0708, was later removed from the sample
} based upon additional observations (see Section 2.3 for details). 
Table 1

X-ray Observation Log

\begin{tabular}{|c|c|c|c|c|c|}
\hline Object Name (SDSS J) & $z^{\mathrm{a}}$ & $\begin{array}{l}\Delta_{\text {Opt-X }}{ }^{b} \\
(\operatorname{arcsec})\end{array}$ & $\begin{array}{l}\text { Observation } \\
\text { Date } \\
\end{array}$ & $\begin{array}{c}\text { Observation } \\
\text { ID }\end{array}$ & $\begin{array}{c}\text { Exp. Time } \\
(\mathrm{ks}) \\
\end{array}$ \\
\hline \multicolumn{6}{|c|}{ Chandra Cycle 11 Objects } \\
\hline $000009.38+135618.4$ & 2.234 & 0.4 & 2009 Aug 15 & 11490 & 9.5 \\
\hline $084842.63+540808.2$ & 2.225 & $\ldots$ & $2009 \operatorname{Dec} 27$ & 11492 & 13.1 \\
\hline $090312.22+070832.4$ & 2.224 & 0.5 & 2009 Dec 29 & 11491 & 11.5 \\
\hline $094602.31+274407.0$ & 2.382 & 0.1 & 2010 Jan 16 & 11489 & 5.0 \\
\hline $112551.88+502803.6$ & 2.200 & 0.1 & 2010 Sep 22 & 11495 & 9.8 \\
\hline $123035.46+332000.5$ & 2.287 & $\ldots$ & $2010 \mathrm{Jul} 20$ & 11493 & 5.3 \\
\hline $145453.53+032456.8$ & 2.367 & 0.0 & 2009 Dec 19 & 11494 & 6.3 \\
\hline $161801.71+070450.2$ & 2.235 & 0.2 & 2010 Jan 1 & 11496 & 7.7 \\
\hline \multicolumn{6}{|c|}{ Archival X-ray Data Objects } \\
\hline $121946.20+124454.1$ & 2.233 & $\ldots$ & $2008 \mathrm{Feb} 14$ & 8039 & 5.1 \\
\hline $123042.52+204941.3$ & 2.280 & $\ldots$ & $2001 \mathrm{Jul} 1$ & 0112650101 & $23.9^{\mathrm{d}}$ \\
\hline $152156.48+520238.5^{\mathrm{e}}$ & 2.190 & 0.1 & $2006 \mathrm{Jul} 16$ & 6808 & 4.1 \\
\hline
\end{tabular}

Notes.

${ }^{\text {a }}$ Redshift for each source, adopted as the $z_{q}$ value in Table 2 .

${ }^{\mathrm{b}}$ Angular distance between the optical and X-ray positions; no entry indicates no X-ray detection.

${ }^{\mathrm{c}}$ The Chandra and XMM-Newton exposure times are corrected for detector dead time.

${ }^{d}$ The MOS exposure time is listed here. The exposure time for the pn detector was $17.5 \mathrm{ks}$.

${ }^{\mathrm{e}}$ This object was discussed in Just et al. (2007).

Table 2

Quasar Redshifts and Narrow Absorption-line Properties

\begin{tabular}{|c|c|c|c|c|}
\hline Object Name (SDSS J) & $z_{\mathrm{CAS}^{\mathrm{a}}}$ & $z_{q}{ }^{\mathrm{b}}$ & $z_{\mathrm{HW}}{ }^{\mathrm{c}}$ & Redshifts of Absorption Systems \\
\hline $000009.38+135618.4$ & $2.2342 \pm 0.0015$ & $2.2342 \pm 0.0015$ & $2.2405 \pm 0.0015$ & 0.9635 \\
\hline $084842.63+540808.2$ & $2.2252 \pm 0.0030$ & $2.2252 \pm 0.0030$ & $2.2065 \pm 0.0017$ & $2.228^{\mathrm{d}}$ \\
\hline $090312.22+070832.4$ & $2.2237 \pm 0.0020$ & $2.2237 \pm 0.0020$ & $2.2145 \pm 0.0018$ & $2.254^{\mathrm{d}, \mathrm{e}}, 1.756$ \\
\hline $094602.31+274407.0$ & $2.3823 \pm 0.0007$ & $2.3823 \pm 0.0007$ & $2.3828 \pm 0.0007$ & $2.323,1.926,1.029$ \\
\hline $112551.88+502803.6$ & $2.1758 \pm 0.0014$ & $2.200 \pm 0.005$ & $2.1778 \pm 0.0015$ & $1.136,1.0825$ \\
\hline $121946.20+124454.1$ & $2.1813 \pm 0.0027$ & $2.2333 \pm 0.015$ & $2.1497 \pm 0.0013$ & $2.2333^{\mathrm{d}}, 2.2086^{\mathrm{d}}, 1.767,1.631$ \\
\hline $123035.46+332000.5$ & $2.2865 \pm 0.0017$ & $2.2865 \pm 0.0017$ & $2.3057 \pm 0.0010$ & $0.928,0.7675$ \\
\hline $123042.52+204941.3$ & $2.2249 \pm 0.0018$ & $2.280 \pm 0.015$ & $\ldots$ & $2.289^{\mathrm{d}}, 2.28^{\mathrm{d}}, 0.727,0.618$ \\
\hline $145453.53+032456.8$ & $2.3672 \pm 0.0013^{\mathrm{f}}$ & $2.3672 \pm 0.0013$ & $2.3613 \pm 0.0023$ & $2.302,1.5245,0.789$ \\
\hline $152156.48+520238.5$ & $2.208 \pm 0.011$ & $2.190 \pm 0.011$ & $2.1648 \pm 0.0017$ & $1.875,1.616$ \\
\hline $161801.71+070450.2$ & $2.2347 \pm 0.0019$ & $2.2347 \pm 0.0019$ & $2.2195 \pm 0.0021$ & $2.1135,2.075$ \\
\hline
\end{tabular}

Notes.

${ }^{a}$ Redshifts from the SDSS CAS.

b Adopted redshifts for the PHL 1811 analogs.

${ }^{\mathrm{c}}$ Redshifts from Hewett \& Wild (2010).

${ }^{\mathrm{d}}$ Associated narrow absorption lines (defined as $|v|<5000 \mathrm{~km} \mathrm{~s}^{-1}$ ).

e This system was only noticed in the HET spectrum (see Section 2.3).

${ }^{\mathrm{f}}$ Average for the CAS redshifts of the two independent spectra of this object: $z=2.3660 \pm 0.0019$ (MJD 52029) and $z=2.3683 \pm 0.0017$ (MJD 52045).

the spectrum for PHL 1811 from the Hubble Space Telescope (HST; Leighly et al. 2007a) are also included in Figure 2. Nine of the 11 objects are radio-quiet $(R<10$; see Section 3 for the definition of $R$ ). $\mathrm{J} 1454+0324$ and $\mathrm{J} 1618+0704$ are radiointermediate with $R=12.8$ and $R=35.0$, respectively. Three of the 11 objects already had sensitive archival X-ray coverage (J1219+1244 and $\mathrm{J} 1521+5202$ by Chandra, $\mathrm{J} 1230+2049$ by XMM-Newton). We proposed short (5-13 ks) Chandra observations for the other eight objects, and were awarded observing time in Cycle 11 (see Table 1 for an X-ray observation log).

\subsection{Measurements of Redshifts and Rest-frame UV Emission-line Properties}

The redshifts and their uncertainties (see Table 2) for our PHL 1811 analogs are the spectro1d redshifts from the SDSS CAS in most cases. These redshifts were obtained by fitting the peaks in the SDSS spectra with a set of strong quasar emission lines (see Section 4.2.10.1 of Stoughton et al. 2002 for details). The SDSS CAS redshift measurements may not be precise for our sample because of the weak, blueshifted, and asymmetric emission lines. We therefore examined the redshift values for our PHL 1811 analogs carefully. For four quasars whose CAS redshifts were suspect based on this examination, we adopt redshifts and uncertainties from other approaches: for J1125+4028, the redshift of the longer-wavelength peak of the double-peaked Mg II emission is adopted as systemic; for both J1219+1244 and J1230+2049, we adopt the redshift of a strong (but not broad) absorption system; for $\mathrm{J} 1521+5202$, we follow Just et al. (2007) and adopt $z=2.19$ based on Mg II emission. The differences between these updated redshift values and the CAS values have a magnitude of $\Delta z=0.02-0.05$. The final redshift values adopted are listed as $z_{q}$ in Table 2 . Redshifts of narrow absorption-line systems (NALs) are also 
Table 3

Quasar UV Spectral Properties

\begin{tabular}{|c|c|c|c|c|c|c|c|c|c|c|}
\hline Object Name (SDSS J) & MJD & C IV Blueshift & C IV FWHM & C IV $\sigma_{\text {line }}$ & C IV FWHM $/ \sigma_{\text {line }}$ & $W_{r}\left(\mathrm{C}_{\text {IV }}\right)$ & $\bar{W}_{r}(\mathrm{Si} \text { IV })^{\mathrm{a}}$ & $W_{r}(\lambda 1900 \AA)^{\mathrm{b}}$ & $\bar{W}_{r}(\mathrm{Fe}$ III $)$ & $\alpha_{v}{ }^{\mathrm{c}}$ \\
\hline $000009.38+135618.4$ & 52235 & $-5200 \pm 1000$ & $6400 \pm 500$ & $4800 \pm 800$ & $1.33 \pm 0.25$ & $5.0 \pm 1.5$ & $2.8 \pm 1.5$ & $4.4 \pm 1.8$ & $2.8 \pm 1.2$ & -0.97 \\
\hline $084842.63+540808.2$ & 51899 & $-4600 \pm 500$ & $7000 \pm 500$ & $5600 \pm 800$ & $1.25 \pm 0.20$ & $10.3 \pm 1.8$ & $4.4 \pm 1.2$ & $11.0 \pm 2.4$ & $5.0 \pm 1.8$ & -0.50 \\
\hline $090312.22+070832.4$ & 52674 & $-2300 \pm 600$ & $6900 \pm 600$ & $2900 \pm 500$ & $2.38 \pm 0.46$ & $6.5 \pm 1.8$ & $2.9 \pm 1.5$ & $8.5 \pm 2.4$ & $6.2 \pm 1.8$ & -0.49 \\
\hline $090312.22+070832.4$ (HET) & 55506 & $-610 \pm 300$ & $6600 \pm 690$ & $3240 \pm 680$ & $2.03 \pm 0.45$ & $5.2 \pm 0.7$ & $3.8 \pm 0.8$ & $9.5 \pm 0.7$ & $6.2 \pm 0.7$ & . \\
\hline $094602.31+274407.0$ & 53385 & $-1700 \pm 500$ & $11000 \pm 800$ & $5800 \pm 800$ & $1.90 \pm 0.30$ & $6.0 \pm 0.9$ & $4.2 \pm 0.6$ & $7.3 \pm 0.9$ & $6.8 \pm 0.9$ & -0.68 \\
\hline $112551.88+502803.6$ & 52365 & $-3400 \pm 700$ & $7400 \pm 600$ & $3500 \pm 600$ & $2.11 \pm 0.40$ & $7.3 \pm 1.8$ & $6.9 \pm 1.8$ & $8.8 \pm 2.1$ & $3.8 \pm 1.5$ & -0.53 \\
\hline $121946.20+124454.1$ & 53115 & $-9400 \pm 300$ & $7100 \pm 500$ & $7500 \pm 1000$ & $0.95 \pm 0.14$ & $8.1 \pm 0.6$ & $3.4 \pm 0.6$ & $8.3 \pm 0.9$ & $7.7 \pm 0.6$ & -0.87 \\
\hline $123035.46+332000.5$ & 53472 & $-2800 \pm 600$ & $11000 \pm 800$ & $5600 \pm 800$ & $1.96 \pm 0.31$ & $10.4 \pm 1.2$ & $4.0 \pm 0.9$ & $12.2 \pm 1.2$ & $6.8 \pm 1.2$ & -0.32 \\
\hline $123042.52+204941.3$ & 54480 & $-5700 \pm 800$ & $4900 \pm 300$ & $8600 \pm 1200$ & $0.57 \pm 0.09$ & $6.8 \pm 1.5$ & $2.2 \pm 1.2$ & $3.5 \pm 1.5$ & $5.6 \pm 1.2$ & -0.88 \\
\hline $145453.53+032456.8^{d}$ & 52037 & $-1600 \pm 600$ & $5500 \pm 500$ & $3900 \pm 600$ & $1.41 \pm 0.25$ & $5.1 \pm 1.3$ & $3.2 \pm 2.1$ & $5.2 \pm 1.2$ & $7.2 \pm 1.4$ & -0.28 \\
\hline $152156.48+520238.5$ & 52376 & $-4900 \pm 300$ & $11700 \pm 800$ & $5700 \pm 800$ & $2.05 \pm 0.32$ & $9.1 \pm 0.6$ & $2.7 \pm 0.3$ & $8.2 \pm 0.6$ & $7.1 \pm 0.6$ & -0.61 \\
\hline $161801.71+070450.2$ & 53884 & $-800 \pm 600$ & $7700 \pm 500$ & $5900 \pm 800$ & $1.31 \pm 0.20$ & $8.3 \pm 1.5$ & $3.5 \pm 1.2$ & $10.1 \pm 1.5$ & $3.9 \pm 1.2$ & -0.38 \\
\hline PHL 1811 & $\ldots$ & $-1400 \pm 250$ & $4300 \pm 700$ & $3400 \pm 1100$ & $1.26 \pm 0.46$ & $4.7 \pm 0.9$ & $4.8 \pm 0.9$ & $8.3 \pm 0.6$ & $4.7 \pm 0.6$ & -0.74 \\
\hline Average $^{\mathrm{e}}$ & $\ldots$ & $-3650 \pm 2450$ & $7600 \pm 2400$ & $5300 \pm 1700$ & $1.44 \pm 0.65$ & $7.3 \pm 2.0$ & $3.8 \pm 1.3$ & $8.0 \pm 2.6$ & $5.6 \pm 1.6$ & -0.60 \\
\hline V01 composite ${ }^{f}$ & $\ldots$ & $-570 \pm 30$ & $5050 \pm 550$ & $4500 \pm 1000$ & $1.12 \pm 0.28$ & $30.0 \pm 0.3$ & $8.7 \pm 0.3$ & $21.7 \pm 0.2$ & $2.9 \pm 0.1$ & -0.44 \\
\hline Y04 composite B4 & $\ldots$ & $-470 \pm 260$ & $4380 \pm 250$ & $4250 \pm 240$ & $1.03 \pm 0.08$ & $26.0 \pm 0.3$ & $8.2 \pm 0.3$ & $22.4 \pm 0.2$ & $3.1 \pm 0.1$ & -0.42 \\
\hline PHL $1092^{\mathrm{h}}$ & $\ldots$ & $-3900 \pm 500$ & $8740 \pm 1080$ & $2690 \pm 700$ & $3.25 \pm 0.29$ & $7.2 \pm 1.2$ & $3.9 \pm 0.9$ & $15.4 \pm 1.2$ & $3.3 \pm 1.2$ & -0.70 \\
\hline
\end{tabular}

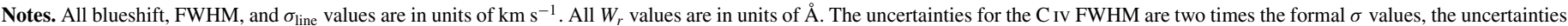

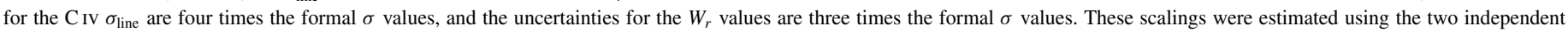
spectra of J1454+0324; each scaling is the rounded ratio of the rms of the two independent measurements and the average of their formal $\sigma$ values.

${ }^{a}$ This line is a blend of Si IV and O IV]; we refer to it as Si IV simply for convenience.

b Mainly C III] $\lambda 1909$, but also including [Ne III] $\lambda 1814$, Si II $\lambda 1816$, Al III $\lambda 1857$, Si III] $\lambda 1892$, and several Fe III multiplets (see Table 2 of Vanden Berk et al. 2001 ).

c The spectral index of the presumed power-law continuum, where $f_{v} \propto v^{\alpha_{\nu}}$

${ }^{\mathrm{d}}$ All the spectral parameters for J1454+0324, including MJD, are the average of two spectra taken at MJD=52029 and MJD=52045.

e The average values for the PHL 1811 analogs plus PHL 1811 itself.

${ }^{f}$ The composite spectrum from Vanden Berk et al. (2001).

g The "B4" composite spectrum from Yip et al. (2004).

h We measured the emission-line properties for PHL 1092 using its HST STIS spectrum (Leighly et al. 2007a). See footnote "20" for further discussion.

listed in Table 2. Three PHL 1811 analogs (J0848+5408, $\mathrm{J} 1219+1244$, and $\mathrm{J} 1230+2049$ ) have associated NALs (defined as $|v|<5000 \mathrm{~km} \mathrm{~s}^{-1}$ ), which are labeled in Figure 2 and in Table 2. The other NALs with $v<-5000 \mathrm{~km} \mathrm{~s}^{-1}$ are most likely intervening absorption.

Hewett \& Wild (2010) reported improved redshift measurements with lower systematic uncertainties for SDSS quasars. Their measurements for our candidate PHL 1811 analogs are also included in Table 2, denoted as $z_{\mathrm{HW}}$. These redshifts are either consistent with or slightly lower than $z_{q}$. However, the $z_{\text {HW }}$ values were determined via cross-correlation with quasar spectral templates. Given the unusual spectral properties of our candidate PHL 1811 analogs, we have not adopted the $z_{\mathrm{HW}}$ values. Nevertheless, we will test whether adopting these different redshift values would affect our main conclusions in the following sections, particularly regarding C IV blueshifts.

We measured emission-line properties interactively for C IV, Si IV, the $\lambda 1900$ complex, ${ }^{12}$ and Fe III UV48 (Table 3), since the automatic measurements stored in the CAS may be unreliable because of the weak, highly blueshifted lines of PHL 1811 analogs. We first fitted the continuum for each source following the method of Vanden Berk et al. (2001). The SDSS spectra were smoothed with a five-pixel sliding-box filter. Regions of strong narrow absorption were interpolated across manually. The fitting region for each emission line was then defined between lower and upper wavelength limits $\lambda_{\text {lo }}$ and $\lambda_{\text {hi }}$ (see Table 2 of Vanden Berk et al. 2001). A power-law local continuum was fit to the lower and upper $10 \%$ of the wavelength region between $\lambda_{\text {lo }}$ and $\lambda_{\text {hi }}$. After subtracting the continuum, we measured $W_{r}$, FWHM, the line dispersion ( $\sigma_{\text {line }}$, the second moment relative to the flux-weighted mean wavelength of the line), and the associated uncertainties for each line. Blueshifts were calculated between 12 Mainly C III] $\lambda 1909$, but also including other features; see footnote $b$ of
Table 3 . the lab wavelength of a line in the quasar rest frame (see Table 2 of Vanden Berk et al. 2001) and the observed mode of all pixels with heights greater than $50 \%$ of the peak height of this line, where mode $=3 \times$ median $-2 \times$ mean. In addition to the C IV blueshift errors quoted in Table 3 , the uncertainties in the redshift measurements also produce further uncertainties for the C IV blueshift. We also include corresponding measurements of the spectrum of PHL 1811 (Leighly et al. 2007a), the composite quasar spectrum of Vanden Berk et al. (2001), the "B4" composite spectrum of high-redshift $(2.06<z<3.33)$, high-luminosity $\left(-28<M_{i}<-26\right)$ quasars of Yip et al. (2004), and the spectrum of PHL 1092 (Leighly et al. 2007a) in Table 3 for comparison.

Using our refined $W_{r}$ values, all our targets and PHL 1811 have $W_{r}(\mathrm{C}$ IV $)<11 \AA, W_{r}(\mathrm{Si}$ IV $)<8 \AA, 3.5 \AA<W_{r}(\lambda 1900 \AA)$ $<12.5 \AA$, and $2.5 \AA<W_{r}\left(\mathrm{Fe}_{\mathrm{III}}\right)<8 \AA$. Our targets have significantly weaker $\mathrm{C}$ IV and SiIV lines than the required selection criteria. For PHL 1811 analogs, the first three of these lines have average $W_{r}$ values that are only $\approx 40 \%$ of the average for normal quasars of similar redshift and luminosity. Therefore, the sources in our sample have weak high-ionization lines and semi-forbidden lines like PHL 1811. In contrast, Fe III UV48 has a 50\% larger average $W_{r}$ in PHL 1811 analogs. As compared to PHL 1811, our PHL 1811 analogs have lines that are just as weak but are typically broader (perhaps partly because our sources are more luminous and thus likely have SMBHs of higher mass; e.g., Laor 2000) and more blueshifted.

Some key emission lines in the rest-frame optical band, e.g., $\mathrm{H} \alpha, \mathrm{H} \beta$, and [O III] $\lambda 5007$, are not covered by the SDSS spectra of our PHL 1811 analogs due to their high redshifts. PHL 1811 has a narrow-line type 1 optical spectrum as well as very weak [O III] emission compared to typical type 1 quasars (Leighly et al. 2007a). Near-infrared spectroscopy of our PHL 1811 analogs is needed to measure the properties of these emission lines. We report near-infrared spectroscopy 


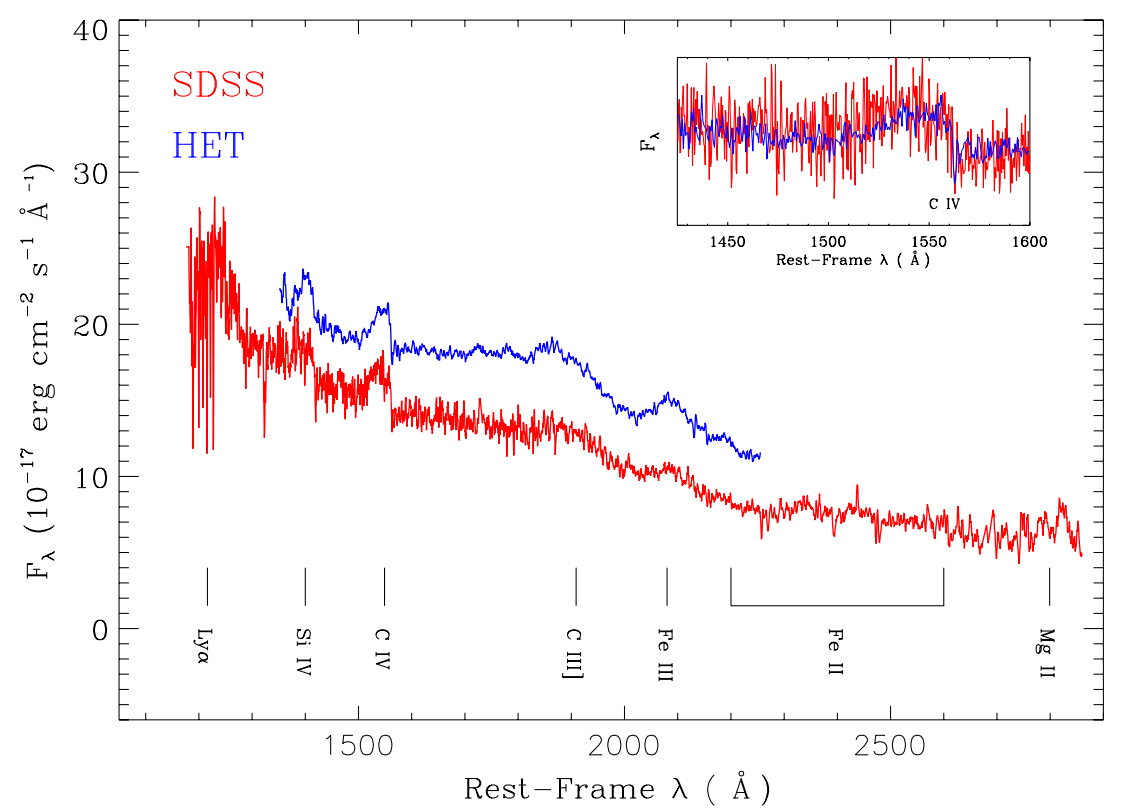

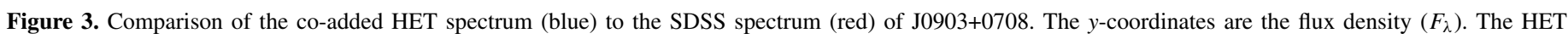

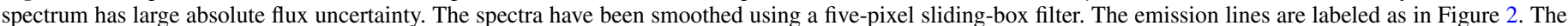

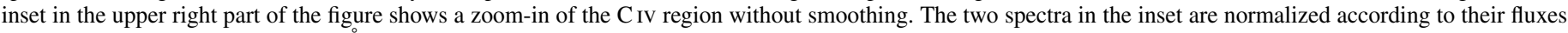
in the wavelength range $1425-1450 \AA$. The resolution of the SDSS spectrum is $R \approx 2000$, while that of the HET spectrum is $R \approx 1000$.

(A color version of this figure is available in the online journal.)

of our most luminous PHL 1811 analog, J1521+5202, in the Appendix of this work.

\subsection{J0903+0708: Not a PHL 1811 Analog}

One radio-quiet source in our sample, J0903+0708, shows notably different $\mathrm{X}$-ray properties from the other radio-quiet sources; it is much brighter in X-rays (see Section 3). After the Chandra observations, we observed this source with the Hobby-Eberly Telescope (HET; Ramsey et al. 1998) because it was a potential outlier. We obtained spectroscopy for J0903+0708 using the G2 grating and the $1^{\prime \prime}$ slit of the Low-Resolution Spectrograph (Hill et al. 1998) on the HET on 2010 November 5. Two HET spectra with spectral resolutions $R \approx 1000$ were extracted, each of which has an exposure time of $600 \mathrm{~s}$. The spectra were calibrated to a standard star (G191 B2B). Figure 3 shows the co-added HET spectrum and a comparison to the SDSS spectrum. The rest-frame wavelength range of the HET spectra is 1350-2250 $\AA$. The rest-frame UV emission-line measurements of the co-added HET spectrum are also listed in Table 3. The strengths of the emission lines ( C IV, Si IV, C III], and Fe III) in the HET spectrum agree with those of the SDSS spectrum, while the blueshift of the C IV line in the HET spectrum is $-610 \pm 300 \mathrm{~km} \mathrm{~s}^{-1}$, different at a $2.5 \sigma$ level from the SDSS measurement $\left(-2300 \pm 600 \mathrm{~km} \mathrm{~s}^{-1}\right)$. In order to test for any possible variation of the $\mathrm{C}$ IV line, we performed a $\chi^{2}$ test on the C IV region (1486-1574 $\AA$ ) of the two spectra. These two spectra are consistent with each other within the noise ( $\chi^{2}=132.3$ for 146 spectral bins). The HET spectrum has a much higher signal-to-noise ratio than the SDSS spectrum (see the inset of Figure 3 for the C IV line region), which indicates that the SDSS blueshift measurement was probably erroneous due to the SDSS spectrum's limited signal-to-noise ratio. With the improved HET data, J0903+0708 would not have passed our original selection criterion of C IV blueshift for PHL 1811 analogs (see Section 2.1), and thus it should not be included in our sample. Note that J0903+0708 was one of the lowest C IV blueshift sources among our candidate PHL 1811 analogs even with only the SDSS measurements. J0903+0708 is probably a regular WLQ (see Section 4.6 for further discussions on the relation between PHL 1811 analogs and WLQs). We will therefore exclude this source from discussion hereafter unless noted. Nevertheless, we will report the X-ray properties of J0903+0708 in Section 3. Our final sample of PHL 1811 analogs includes 10 quasars; 8 are radio quiet, while 2 are radio intermediate.

\subsection{Measurements of the UV Continua}

We also estimated the spectral index of the presumed powerlaw continuum in the rest-frame 1200-2800 $\AA$ range $\left(\alpha_{\nu}\right.$, where $\left.f_{v} \propto v^{\alpha_{v}}\right)$ for each source by fitting the SDSS spectra in four line-free regions using the method described in Section 2.1 of Gibson et al. (2008b). The results are listed in Table 3. The range of spectral indices for our candidate PHL 1811 analogs is -0.97 to -0.28 with a mean value of $\left\langle\alpha_{v}\right\rangle=-0.61$. This value is within the range seen for typical quasars, although it is somewhat steeper (i.e., redder) than the "canonical" value of $\alpha_{v}=-0.5$ the optical and UV region because of the presence of the "small blue bump" due to the Balmer continuum and $\mathrm{Fe}$ emission (see discussion in Section 2.1 of Strateva et al. 2005; also see Natali et al. 1998; Schneider et al. 2001; Vanden Berk et al. 2001).

The somewhat redder than average nature of our quasars is evidenced by their $\Delta(g-i)$ values (the $g-i$ color minus the average $g-i$ color for quasars at the same redshift) taken from Schneider et al. (2010). On average, our sample is redder than $\approx 75 \%$ of other quasars at the same redshift, with $\langle\Delta(g-i)\rangle=$ 0.28 . About a quarter of this excess can be attributed to weak CIV emission in the $g$ band and about a quarter to strong Fe II emission in the $i$ band (see Figure 2). The remaining half corresponds to the redder continuum expected for quasars with spectral index $\alpha_{v}=-0.61$ instead of $\alpha_{v}=-0.5$.

In spite of their somewhat redder colors, we do not expect substantial incompleteness in the SDSS selection of PHL 1811 

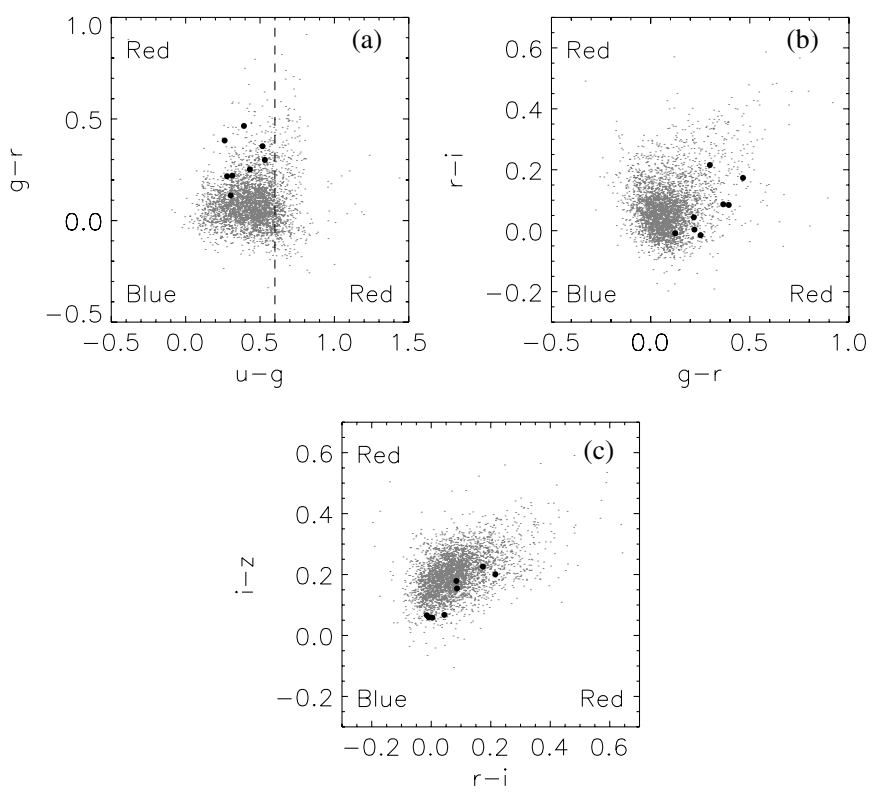

Figure 4. Locations of our PHL 1811 analogs (black filled circles) in the SDSS color space: (a) $u-g$ vs. $g-r$, (b) $g-r$ vs. $r-i$, and (c) $r-i$ vs. $i-z$. The small gray dots show the SDSS color-selected quasars in the DR7 catalog (Schneider et al. 2010) with similar redshifts $(2.125<z<2.385)$ and luminosities $\left(M_{i}>-26.5\right)$. The dashed line in panel (a) shows an inclusion region $(u-g)<0.6$ of the SDSS color selection for quasar candidates.

analogs. Our PHL 1811 analogs lie within the loci of $u-g, g-r$, $r-i$, and $i-z$ colors for SDSS color-selected quasars at similar redshifts and luminosities, but they are somewhat offset from the regions with the highest density of quasars (see Figure 4). They are within the inclusion region of $(u-g)<0.6$ for SDSS color selection of quasar candidates (see Section 3.5.2 of Richards et al. 2002). Our objects are also in a redshift range where the SDSS color selection algorithm has high completeness $(\approx 75 \%$; see Figure 6 of Richards et al. 2006).

\section{X-RAY DATA ANALYSIS}

The eight Chandra Cycle 11 targets were observed with the S3 CCD of the Advanced CCD Imaging Spectrometer (ACIS; Garmire et al. 2003). Data reduction was performed using the standard CIAO v4.2 procedures. X-ray images were generated for the observed-frame soft $(0.5-2.0 \mathrm{keV})$, hard $(2.0-8.0 \mathrm{keV})$, and full (0.5-8.0 keV) bands using ASCA grade 0, 2, 3, 4, and 6 events. Source detections were performed with the WAVDETECT algorithm (Freeman et al. 2002) using a detection threshold of $10^{-5}$ and wavelet scales of $1, \sqrt{2}, 2,2 \sqrt{2}$, and 4 pixels. All targets, except $\mathrm{J} 0848+5408$ and $\mathrm{J} 1230+3320$, were detected in at least one band within 0.'5 of the object's optical coordinates. Aperture photometry was performed using the IDL APER routine with an aperture radius of $1^{\prime \prime} .5$ for the source $(\approx 95 \%$ enclosed energy for soft band, $\approx 90 \%$ enclosed energy for hard band), and inner and outer annulus radii of 3.'0 and $4^{\prime \prime} .5$ for background subtraction, respectively. All background regions are free of $\mathrm{X}$-ray sources. For undetected sources, the upper limits upon X-ray counts were determined using the method of Kraft et al. (1991) for the low-count scenario of $N<10$, where $N$ is the total counts within the source aperture. Aperture corrections were applied to the X-ray counts or to their upper limits. The $\mathrm{X}$-ray counts in the three bands defined above, as well as the band ratio (defined as the hard counts divided by the soft counts) and effective power-law photon index, for all newly observed and archival sources, are reported in Table 4. The effective power-law photon index was calculated from the band ratio using the Chandra PIMMS $^{13}$ tool, assuming a power-law model with Galactic absorption.

One archival source, J1521+5202, was observed by Chandra in Cycle 7; these results were reported in Just et al. (2007). We reanalyzed these Chandra data and found consistent results with those in Just et al. (2007). This extremely optically luminous $\left(M_{i}=-30.19\right.$, see Figure 1$)$ source is exceptionally X-ray weak; it is not detected in the soft band. The other Chandra archival source, J1219+1244, was serendipitously covered in Cycle 8, with an off-axis angle of 3.0. It is undetected in all three bands. The aperture for this source ( 2 .' 1 radius) was taken to be the $95 \%$ enclosed-energy radius at $1.497 \mathrm{keV}$ according to the point-spread function (PSF) of the ACIS-S detector.

The XMM-Newton archival source, J1230+2049, was serendipitously observed on 2001 July 1 . The data were processed using standard XMM-Newton Science Analysis System (v10.0.0) routines. Only data from the MOS detectors were used because this source is located on a CCD edge of the pn detector. The event files were filtered by removing periods of background flaring in which the count rate exceeded $0.35 \mathrm{~s}^{-1}$ for events with energies above $10 \mathrm{keV}$. Only $3 \%$ of the exposure time was removed via this filtering procedure. This source is undetected in all three bands using the EBOXDETECT procedure. The aperture for photometry ( 9 '. 3 radius) was taken to be the $50 \%$ enclosed-energy radius (to avoid a nearby X-ray source) at $1.5 \mathrm{keV}$ according to the PSF of the MOS detectors at an offaxis angle of 6'0. The upper limits upon X-ray counts were also determined using the Kraft et al. (1991) method.

Table 5 lists the key X-ray, optical, and radio properties of our sample.

Column (1) gives the SDSS J2000 equatorial coordinates for the quasar.

Column (2) contains the apparent $i$-band magnitude of the quasar using the SDSS quasar catalog BEST photometry, $m_{i}$.

Column (3) is the absolute $i$-band magnitude for the quasar, $M_{i}$, from the SDSS DR7 quasar catalog (Schneider et al. 2010), which was calculated by correcting for Galactic extinction and assuming a power-law spectral index of $\alpha_{v}=-0.5$.

Column (4) provides the Galactic neutral hydrogen column density obtained with the Chandra COLDEN ${ }^{14}$ tool (Dickey \& Lockman 1990; Stark et al. 1992) in units of $10^{20} \mathrm{~cm}^{-2}$.

Column (5) gives the count rate in the observed-frame soft $\mathrm{X}$-ray band $(0.5-2.0 \mathrm{keV})$ in units of $10^{-3} \mathrm{~s}^{-1}$. For the two offaxis sources (J1219+1244 and J1230+2049), the upper limits on count rate are corrected for vignetting using exposure maps.

Column (6) contains the Galactic absorption-corrected flux in the observed-frame soft X-ray band $(0.5-2.0 \mathrm{keV})$ obtained with Chandra PIMMS tool, in units of $10^{-14} \mathrm{erg} \mathrm{cm}^{-2} \mathrm{~s}^{-1}$. An absorbed power-law model was used with a photon index $\Gamma=2$, which is typical for quasars, and the Galactic neutral hydrogen column density $\left(N_{H}\right.$, given in Column 4$)$.

Column (7) provides the Galactic absorption-corrected flux density at rest-frame $2 \mathrm{keV}$ obtained with PIMMS, in units of $10^{-32} \mathrm{erg} \mathrm{cm}^{-2} \mathrm{~s}^{-1} \mathrm{~Hz}^{-1}$.

Column (8) gives the logarithm of the Galactic absorptioncorrected quasar luminosity in the rest-frame $2-10 \mathrm{keV}$ band.

Column (9) is the continuum flux density at rest-frame $2500 \AA$ in units of $10^{-27} \mathrm{erg} \mathrm{cm}^{-2} \mathrm{~s}^{-1} \mathrm{~Hz}^{-1}$, which was obtained

\footnotetext{
13 http://cxc.harvard.edu/toolkit/pimms.jsp

14 http://cxc.harvard.edu/toolkit/colden.jsp
} 
Table 4

X-ray Counts

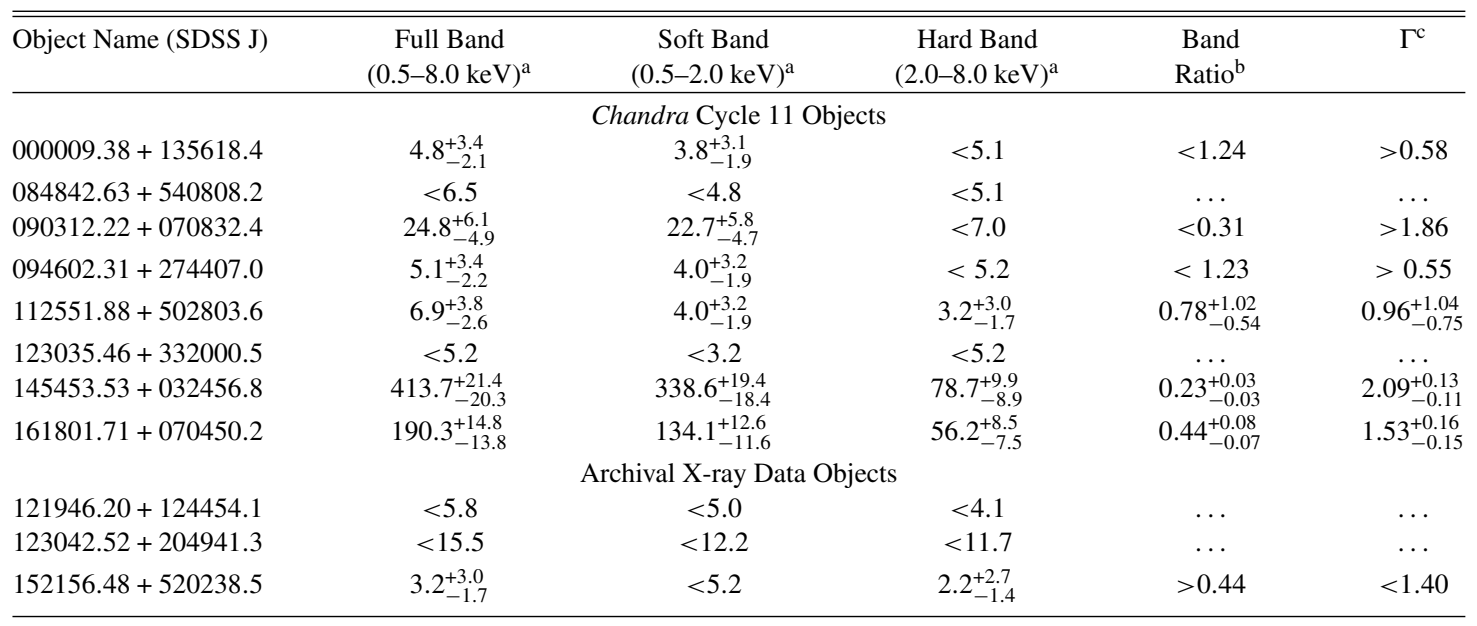

Notes.

${ }^{\text {a }}$ Errors on the X-ray counts were calculated using Poisson statistics corresponding to the $1 \sigma$ significance level according to Tables 1 and 2 of Gehrels (1986).

$\mathrm{b}$ The band ratio is defined here as the number of hard-band counts divided by the number of soft-band counts. The errors on the band ratio correspond to the $1 \sigma$ significance level and were calculated using Equation (1.31) in Section 1.7.3 of Lyons (1991). The band ratios for all of the Chandra objects observed in the same cycle can be directly compared with one another.

${ }^{\mathrm{c}}$ The effective power-law photon indices were calculated using the Chandra PIMMS tool (version 3.9k). The effect of the quantum efficiency decay over time at low energies of the ACIS detector were corrected for Chandra observed objects.

Table 5

X-ray and Optical Properties

\begin{tabular}{|c|c|c|c|c|c|c|c|c|c|c|c|c|c|}
\hline Object Name (SDSS J) & $\begin{array}{l}m_{i} \\
\text { (2) } \\
\end{array}$ & $\begin{array}{l}M_{i} \\
\text { (3) } \\
\end{array}$ & $N_{\mathrm{H}}$ & $\begin{array}{c}\text { Count } \\
\text { Rate }^{\mathrm{a}} \\
(5) \\
\end{array}$ & $\begin{array}{c}F_{0.5-2 \mathrm{keV}^{\mathrm{b}}} \\
(6)\end{array}$ & $\begin{array}{c}f_{2 \mathrm{keV}} \\
(7) \\
\end{array}$ & $\begin{array}{c}\log L \\
(2-10 \mathrm{keV}) \\
(8)\end{array}$ & $\begin{array}{c}f_{2500 \AA} \\
(9) \\
\end{array}$ & $\begin{array}{c}\log L_{v} \\
(2500 \AA) \\
(10)\end{array}$ & $\begin{array}{l}\alpha_{\mathrm{ox}} \\
(11)\end{array}$ & $\begin{array}{c}\Delta \alpha_{\mathrm{OX}}(\sigma)^{\mathrm{c}} \\
(12) \\
\end{array}$ & $\begin{array}{c}f_{\mathrm{x}-\text { weak }}{ }^{\mathrm{d}} \\
(13)\end{array}$ & $(14)$ \\
\hline \multicolumn{14}{|c|}{ Chandra Cycle 11 Objects } \\
\hline $000009.38+135618.4$ & 18.30 & -27.44 & 3.77 & $0.40_{-0.19}^{+0.33}$ & 0.17 & 0.83 & 43.90 & 2.02 & 31.39 & -2.07 & $-0.38(2.58)$ & 9.77 & $<4.7$ \\
\hline $084842.63+540808.2$ & 18.76 & -26.90 & 3.09 & $<0.37$ & $<0.16$ & $<0.76$ & $<43.85$ & 1.12 & 31.13 & $<-1.98$ & $<-0.33(2.27)$ & $>7.24$ & $<2.6$ \\
\hline $090312.22+070832.4$ & 18.64 & -27.11 & 4.54 & $1.98_{-0.41}^{+0.51}$ & 0.87 & 4.21 & 44.59 & 1.45 & 31.24 & -1.74 & $-0.07(0.50)$ & 1.52 & $<2.4$ \\
\hline $094602.31+274407.0$ & 17.00 & -28.81 & 1.73 & $0.80_{-0.38}^{+0.64}$ & 0.33 & 1.65 & 44.24 & 6.57 & 31.95 & -2.15 & $-0.38(2.61)$ & 9.77 & 4.0 \\
\hline $112551.88+520803.6$ & 18.45 & -27.13 & 1.25 & $0.41_{-0.20}^{+0.33}$ & 0.16 & 0.79 & 43.86 & 1.62 & 31.28 & -2.04 & $-0.36(2.50)$ & 8.67 & $<1.2$ \\
\hline $123035.46+332000.5$ & 17.80 & -27.90 & 1.40 & $<0.60$ & $<0.24$ & $<1.19$ & $<44.07$ & 2.70 & 31.53 & $<-2.06$ & $<-0.35(2.37)$ & $>8.16$ & $<1.0$ \\
\hline $145453.53+032456.8$ & 17.95 & -27.88 & 3.59 & $53.36_{-2.90}^{+3.06}$ & 22.97 & 115.39 & 46.08 & 2.75 & 31.57 & -1.30 & $0.42(2.86)$ & 0.08 & 12.8 \\
\hline $161801.71+070450.2$ & 18.17 & -27.58 & 4.59 & $17.47_{-1.51}^{+1.64}$ & 7.74 & 37.37 & 45.55 & 1.62 & 31.29 & -1.40 & $0.28(1.92)$ & 0.19 & 35.0 \\
\hline \multicolumn{14}{|c|}{ Archival X-ray Data Objects } \\
\hline $\begin{array}{l}121946.20+124454.1 \\
123042.52+204941.3\end{array}$ & 17.90 & -27.75 & $\begin{array}{l}2.65 \\
1.99\end{array}$ & $\begin{array}{l}<0.97 \\
<035\end{array}$ & $<0.37$ & $<1.78$ & $<44.23$ & 3.02 & 31.56 & $<-2.01$ & $<-0.29(2.01)$ & $>5.70$ & $\begin{array}{r}3.9 \\
-18\end{array}$ \\
\hline $123042.52+204941.3$ & 18.45 & -27.22 & 1.99 & $<0.35$ & $<0.27$ & $<1.34$ & $<44.12$ & 1.39 & 31.24 & $<-1.93$ & $-0.26(1.76)$ & $>4.76$ & $<1.8$ \\
\hline $152156.48+520238.5$ & 15.44 & -30.19 & 1.58 & $0.78_{-0.41}^{+0.73}$ & 0.25 & 1.18 & 44.03 & 26.11 & 32.48 & -2.44 & $-0.59(4.53)$ & 34.46 & $<0.1$ \\
\hline
\end{tabular}

Notes. The detailed explanation of each column is given in Section 3.

a The count rate in the observed-frame soft X-ray band $(0.5-2.0 \mathrm{keV})$ in units of $10^{-3} \mathrm{~s}^{-1}$, except for J1521+5202 where the count rate is in the observed-frame full band $(0.5-8.0 \mathrm{keV})$ because this source is undetected in the soft band, but detected in the full band.

${ }^{\mathrm{b}}$ The Galactic absorption-corrected observed-frame flux between 0.5 and $2.0 \mathrm{keV}$ in units of $10^{-14} \mathrm{erg} \mathrm{cm}^{-2} \mathrm{~s}^{-1}$. The value for J1521+5202 is also for the flux between 0.5 and $2.0 \mathrm{keV}$, calculated from the full-band count rate.

${ }^{c} \Delta \alpha_{\mathrm{ox}}$ : the difference between the measured $\alpha_{\mathrm{ox}}$ and the expected $\alpha_{\mathrm{ox}}$, defined by the $\alpha_{\mathrm{ox}}-L_{2500 \AA}$ relation in Equation (3) of Just et al. (2007); the statistical significance of this difference, $\sigma$, is measured in units of the rms for $\alpha_{\text {ox }}$ defined in Table 5 of Steffen et al. (2006).

$\mathrm{d}$ The factor of X-ray weakness compared to a typical quasar with similar optical/UV luminosity; see Section 3.

by fitting the SDSS continuum in line-free regions using the method described in Section 2.1 of Gibson et al. (2008b). The SDSS spectra were corrected for fiber light loss and Galactic extinction.

Column (10) contains the logarithm of the monochromatic luminosity at rest-frame $2500 \AA$, calculated from the flux density at rest-frame $2500 \AA$.

Column (11) provides the X-ray-to-optical power-law slope, given by

$$
\alpha_{\mathrm{OX}}=\frac{\log \left(f_{2 \mathrm{keV}} / f_{2500 \AA}\right)}{\log \left(v_{2 \mathrm{keV}} / \nu_{2500 \AA}\right)}=0.384 \log \left(\frac{f_{2 \mathrm{keV}}}{f_{2500 \AA}}\right) .
$$

All measures of flux density used are per unit frequency. Note our UV and X-ray measurements were not simultaneous.

Column (12) is $\Delta \alpha_{\mathrm{ox}}$, defined as

$$
\Delta \alpha_{\mathrm{ox}}=\alpha_{\mathrm{ox}(\text { measured })}-\alpha_{\mathrm{ox}(\text { expected })} .
$$


Table 6

Results of Two-sample Tests

\begin{tabular}{|c|c|c|c|c|}
\hline Method & \multicolumn{2}{|c|}{ J0903+0708 Excluded } & \multicolumn{2}{|c|}{ J0903+0708 Included } \\
\hline Peto-Prentice & 11.115 & $1.06 \times 10^{-28}$ & 8.779 & $1.65 \times 10^{-18}$ \\
\hline Gehan (Hypergeometric Variance) & 3.959 & $7.53 \times 10^{-5}$ & 4.008 & $6.12 \times 10^{-5}$ \\
\hline logrank & 5.415 & $6.13 \times 10^{-8}$ & 5.334 & $9.61 \times 10^{-8}$ \\
\hline
\end{tabular}

Notes. For the detailed definition of each test statistic, see Feigelson \& Nelson (1985) and references therein; also see the ASURV manual at http://astrostatistics.psu.edu/statcodes/asurv. The null-hypothesis probability was calculated from each test statistic using a Gaussian distribution, e.g., for Peto-Prentice test, $1-P_{G}=1.06 \times 10^{-28}$, where $P_{G}$ is the cumulative Gaussian probability at $11.115 \sigma$.

The expected $\alpha_{\mathrm{ox}}$ value for a typical quasar is calculated from the $\alpha_{\mathrm{ox}}-L_{2500} \AA$ correlation given as Equation (3) of Just et al. (2007). The statistical significance of this difference, given in parentheses, is in units of $\sigma$, which is given in Table 5 of Steffen et al. (2006) as the rms for $\alpha_{\text {ox }}$ for several ranges of luminosity. Here, $\sigma=0.146$ for $31<\log L_{2500 \AA}<32$ and $\sigma=0.131$ for $32<\log L_{2500 \AA}<33$.

Column (13) gives the factor of X-ray weakness, converted from the $\Delta \alpha_{\text {ox }}$ values in Column (12), quantifying the $\mathrm{X}$-ray weakness of our targets compared to a typical quasar with similar UV/optical luminosity, which is calculated as $f_{\mathrm{x} \text {-weak }}=10^{-\Delta \alpha_{\mathrm{ox}} / 0.384} \approx 403^{-\Delta \alpha_{\mathrm{ox}}}$. A source with a $\Delta \alpha_{\mathrm{ox}}$ value of -0.384 has an X-ray flux only $\approx 10 \%$ that of typical quasars, corresponding to an X-ray weakness factor of $\approx 10$.

Column (14) provides the radio-loudness parameter, given by

$$
R=\frac{f_{5 \mathrm{GHz}}}{f_{4400 \AA}} .
$$

The denominator, $f_{4400 \AA}$, was found via extrapolation from $f_{2500 \AA}$ using a UV/optical power-law slope of $\alpha_{v}=-0.5$. We also utilized the individual measurements of $\alpha_{v}$ for each source given in Section 2.4, and we found that these would not change the source classifications as radio quiet $(R<10)$ or radio intermediate $(10<R<100)$. The numerator, $f_{5 \mathrm{GHz}}$, was found using a radio power-law slope of $\alpha_{v}=-0.8$ and a flux at $20 \mathrm{~cm}, f_{20 \mathrm{~cm}}$. For sources detected by the FIRST survey (Faint Images of the Radio Sky at Twenty-Centimeters; Becker et al. 1995), $f_{20 \mathrm{~cm}}$ was taken from the FIRST source catalog. For sources covered but not detected by the FIRST survey, the upper limits for $f_{20 \mathrm{~cm}}$ were calculated as three times the rms noise in a $0.5 \times 0.5$ FIRST image cutout at the object's coordinates. J0000+1356, not located in the FIRST survey area, is covered but not detected by the NVSS (The NRAO VLA Sky Survey; Condon et al. 1998). The upper limit for $f_{20 \mathrm{~cm}}$ for this source is taken as $1.35 \mathrm{mJy}$, which is three times the rms noise of the NVSS. Two sources in our sample, J1454+0324 and $\mathrm{J} 1618+0704$, are radio-intermediate quasars; their X-ray emission may contain significant contributions from associated jets.

\section{RESULTS AND DISCUSSION}

\subsection{Relative X-Ray Brightness}

The $\Delta \alpha_{\text {ox }}$ parameter (see Column 12 of Table 5) quantifies the relative X-ray brightness of a quasar with respect to a typical radio-quiet quasar with the same UV luminosity. Figure 2 shows the SDSS spectra of our PHL 1811 analogs (and also J0903+0708; see Section 2.3) ordered by
$\Delta \alpha_{\text {ox }}$. No strong trends are apparent among the PHL 1811 analogs between $\Delta \alpha_{\mathrm{ox}}$ and the UV emission-line characteristics (see Section 4.5 for quantitative correlation analyses). Two radio-intermediate sources in our sample ${ }^{15}$ are X-ray bright (see further discussion in Section 4.7). The distribution of $\Delta \alpha_{\text {ox }}$ values for the eight radio-quiet PHL 1811 analogs is shown in Figure 5(a), compared to that for the 132 radio-quiet, non-BAL quasars in Sample B of Gibson et al. (2008a). ${ }^{16}$ The Sample B quasars were all serendipitously detected by Chandra (with offaxis angles greater than $1^{\prime}$ ). They are representative of typical SDSS quasars in the redshift range of $1.7 \leqslant z \leqslant 2.7$. We use a Peto-Prentice test (e.g., Latta 1981), implemented in the Astronomy Survival Analysis (ASURV) package (e.g., Lavalley et al. 1992), to assess whether these two samples follow the same distribution. The Peto-Prentice test is our preferred approach because it is the least affected by the factors of unequal sample sizes or different censoring patterns that exist in our case (e.g., Latta 1981). The distribution of $\Delta \alpha_{\mathrm{ox}}$ values for our sample is found to be significantly different from that for typical SDSS quasars; the probability of the null hypothesis (the two samples following the same distribution) is reported to be $1 \times 10^{-28}$ by the Peto-Prentice test. Other two-sample tests for censored data (e.g., Gehan, logrank, and Peto-Peto) give similar results (see Table 6). The two-sample test results do not change materially if J0903+0708 (see Section 2.3) is included (also see Table 6).

The mean value of $\Delta \alpha_{\mathrm{ox}}$ for radio-quiet PHL 1811 analogs in our sample is $-0.423 \pm 0.049$, which is calculated using the Kaplan-Meier estimator ${ }^{17}$ (also implemented in the ASURV package), while the mean $\Delta \alpha_{\text {ox }}$ value for radio-quiet, non-BAL quasars in Sample B of Gibson et al. (2008a) is $-0.001 \pm$ 0.011. Therefore, radio-quiet PHL 1811 analogs in our sample

\footnotetext{
15 "Our sample" here refers to our 10 PHL 1811 analogs.

16 Sample B of Gibson et al. (2008a) includes 139 objects characterized as radio-quiet, non-BAL quasars. However, we have identified a further seven of these sources as BAL quasars (with $\mathrm{C}_{\mathrm{IV} \mathrm{B}} \mathrm{BI}_{0}>0$ ) in the SDSS DR5 BAL quasar catalog (Gibson et al. 2009b; the sources are J0050-0057, $\mathrm{J} 0756+4458, \mathrm{~J} 1212+1520, \mathrm{~J} 1426+3517, \mathrm{~J} 1426+3753, \mathrm{~J} 1430+3221$, and $\mathrm{J} 1434+3340$ ). For four of these seven sources, the classification depends on the reconstruction of the $\mathrm{C}$ IV emission-line profile (see the discussion of the Emlost flag in Section 2 of Gibson et al. 2009b). In the other three cases, BAL classification is sensitive to the continuum model placement. The continuum models in Gibson et al. (2009b) were generally different from those in Gibson et al. (2008a), so the identifications of BALs may differ in these cases. An additional six sources (J0855+3709, J1049+5858, J1245-0021, J1338+2922, $\mathrm{J} 1427+3241$, and $\mathrm{J} 1618+3456$ ) appear to have possible BAL or mini-BAL features in our visual inspections of their SDSS spectra. The seven quantitatively identified BAL quasars are removed in the following analysis. The six visually identified BAL or mini-BAL quasars will be kept in Sample $\mathrm{B}$, but shown by different symbols in subsequent figures.

17 The Kaplan-Meier estimator is applicable to censored data and reliable for
} small-sample cases (E. D. Feigelson 2010, private communication). 

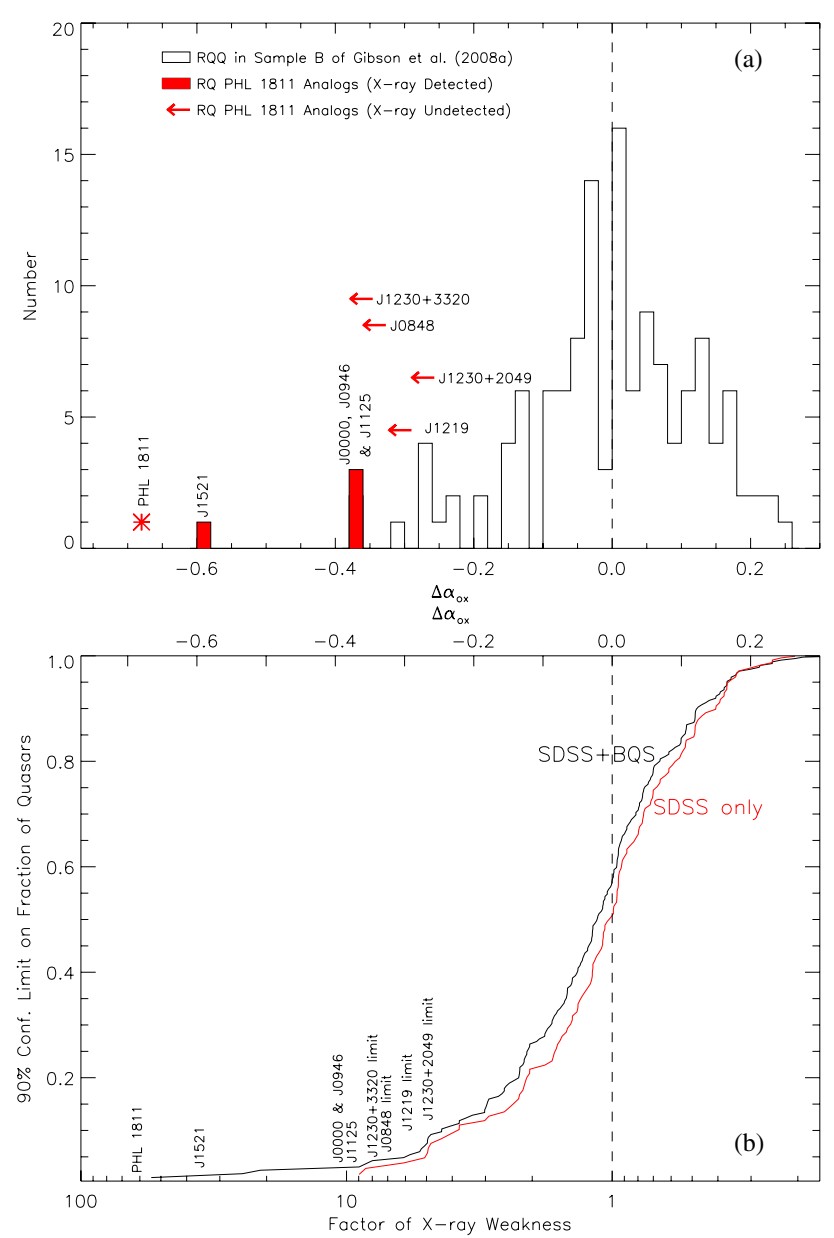

Figure 5. (a) Distribution of $\Delta \alpha_{\text {ox }}$ values for radio-quiet objects $(R<10)$ in our sample of PHL 1811 analogs, compared to that of the 132 radio-quiet, non-BAL quasars in Sample B of Gibson et al. (2008a). The red shaded histogram and leftward arrows represent radio-quiet sources in our sample which are detected and undetected in X-rays, respectively. The unshaded histogram shows the radio-quiet, non-BAL quasars in Sample B of Gibson et al. (2008a). (b) $90 \%$ confidence upper limit on the fraction of SDSS (red curve) and SDSS+BQS (black curve) radio-quiet, non-BAL quasars that are X-ray weak by a given factor (adapted from Figure 5 of Gibson et al. 2008a). The factors of X-ray weakness for PHL 1811 analogs in our sample are shown. The $\Delta \alpha_{\mathrm{ox}}$ values and the corresponding X-ray weakness factors in the two panels are aligned with each other. Source names for PHL 1811 analogs are labeled in both panels in the format of "Jhhmm" for brevity, except for J1230+3320 and J1230+2049. The dashed vertical line in each panel shows $\Delta \alpha_{\mathrm{ox}}=0$, corresponding to an X-ray weakness factor of unity.

(A color version of this figure is available in the online journal.)

are anomalously $\mathrm{X}$-ray weak by a mean factor of $\approx 13$. If $\mathrm{J} 0903+0708$ is included, the mean value of $\Delta \alpha_{\text {ox }}$ changes to $-0.384 \pm 0.057$, corresponding to a mean X-ray weakness factor of $\approx 10$. All of our radio-quiet PHL 1811 analogs are $\mathrm{X}$-ray weak by factors $>4.8$; we only have lower limits on the range of X-ray weakness factors because four of the PHL 1811 analogs are undetected in X-rays. Our X-ray detected sources show that the X-ray weakness factors span at least the range from 8.7 to 34.5 .

\subsection{Average X-Ray Properties via Stacking Analyses}

For the three radio-quiet PHL 1811 analogs not detected by Chandra (J0848+5408, J1219+1244, and J1230+3320), we performed a stacking analysis to obtain an average constraint on their relative X-ray weakness. We added the total counts and background counts of the three sources in their apertures for the full band, soft band, and hard band. For each band, we then calculated the Poisson probability of the total counts arising from a fluctuation of the corresponding background counts. If this probability is less than $0.1 \%$, we take the stacked source to be detected in that band. The stacked source is detected in the full band, with 4.42 total counts and 0.35 background counts; the Poisson probability is $1 \times 10^{-4}$. The stacked source is not detected in the soft or hard band, so a band-ratio analysis is not possible. The combined effective exposure time for the stacked source is $23.3 \mathrm{ks}$. After an aperture correction was applied, the average full-band count rate of the stacked source is $1.84 \times$ $10^{-4}$ counts $\mathrm{s}^{-1}$. We adopted the average values of the parameters for the three sources, such as Galactic $N_{H}, z$, and $f_{2500 \AA}$, to calculate the X-ray properties of the stacked source. Under the assumption of a Galactic-absorbed power-law model with $\Gamma=2$, the average $\mathrm{X}$-ray flux density at rest-frame $2 \mathrm{keV}$ is $2.90 \times 10^{-33} \mathrm{erg} \mathrm{cm}^{-2} \mathrm{~s}^{-1} \mathrm{~Hz}^{-1}$, while the average rest-frame $\mathrm{X}$-ray luminosity from $2-10 \mathrm{keV}$ is $2.75 \times 10^{43} \mathrm{erg} \mathrm{s}^{-1}$. The average $\alpha_{\text {ox }}$ value for the stacked source is -2.26 . The $\Delta \alpha_{\mathrm{ox}}$ value is -0.57 , corresponding to an X-ray weakness factor of 30.5 (cf. Figure 5). At least on average, these undetected PHL 1811 analogs appear to be only a few times X-ray weaker than those we have detected individually. PHL 1811 itself has similar X-ray weakness $(\approx 30-100)$ to these undetected PHL 1811 analogs on average (as does J1521+5202).

We also performed a stacking analysis of the four radio-quiet PHL 1811 analogs that are detected by Chandra (J0000+1356, $\mathrm{J} 0946+2744, \mathrm{~J} 1125+5028$, and $\mathrm{J} 1521+5202$ ) to constrain their average X-ray spectral properties. We added the counts for each source in soft band and hard band. The total backgroundsubtracted counts are $12.9_{-3.5}^{+4.7}$ in the soft band and $7.7_{-2.7}^{+3.9}$ in the hard band. The band ratio is $0.60_{-0.26}^{+0.38}$, corresponding to an effective photon index of $\Gamma=1.22_{-0.45}^{+0.52}$ using the average Galactic neutral hydrogen column density of these four sources. This photon-index value suggests a harder average X-ray spectrum for the X-ray detected, radio-quiet PHL 1811 analogs than that for typical radio-quiet quasars. This average $\mathrm{X}$-ray spectrum is also harder than the X-ray spectrum of PHL 1811 ( $\Gamma \approx 2.2$; Leighly et al. 2007b). However, even the quoted $68 \%$ confidence error bars are large due to limited counts. If quoting $90 \%$ confidence error bars, the photon index is $\Gamma=1.22_{-0.66}^{+0.85}$. If we furthermore add the counts from the three PHL 1811 analogs undetected by Chandra (see the previous paragraph), the average effective photon index becomes $\Gamma=$ $1.10_{-0.40}^{+0.45}\left(68 \%\right.$ confidence level), or $1.10_{-0.59}^{+0.77}(90 \%$ confidence level).

\subsection{Demographic Constraints}

Our sample selection and observational results allow us to investigate the demographics of PHL 1811 analogs in the total quasar population. Our PHL 1811 analogs were selected from high-redshift $(2.125 \leqslant z \leqslant 2.385)$, optically bright $\left(m_{r} \leqslant 18.8\right)$ objects classified as QSO or HIZ_QSO in the SDSS DR7 CAS (see Section 2.1). There are 1358 radio-quiet, non-BAL SDSS DR7 quasars within the above redshift and magnitude ranges. Our sample of PHL 1811 analogs contains eight radio-quiet and two radio-intermediate sources. The radio-quiet PHL 1811 analogs thus appear to be only $8 / 1358=\left(0.6_{-0.3}^{+0.5}\right) \%$ (at the $90 \%$ confidence level) of the total radio-quiet, non-BAL quasar population. Considering the potential incompleteness of our sample selection, we take $0.3 \%$ as a lower limit on the fraction of PHL 1811 analogs in the radio-quiet, non-BAL 
quasar population. On the other hand, Figure 5(b) shows upper limits on the fraction of optically selected, radio-quiet, nonBAL quasars compiled from samples using SDSS only the SDSS+BQS (Bright Quasar Survey; Schmidt \& Green 1983) that are X-ray weak by a given factor (adapted from Figure 5 of Gibson et al. 2008a). Only $\leqslant 2.8 \%$ (at the $90 \%$ confidence level) of those quasars are as X-ray weak $\left(\Delta \alpha_{\mathrm{ox}}=-0.427\right.$; see Section 4.1) as the radio-quiet sources in our sample. In summary, the fraction of radio-quiet PHL 1811 analogs should be within the range of $0.3 \%-2.8 \%$. The upper limit of $2.8 \%$ is obtained solely based on the factor of X-ray weakness. Since our selection of PHL 1811 analogs should not have strong incompleteness (a factor of $\lesssim 2$ ), we estimate the fraction of PHL 1811 analogs in the total quasar population to be $\lesssim 1.2 \%$.

Radio-quiet PHL 1811 analogs, which are all X-ray weak in our sample, only compose a small fraction of the total quasar population. They do not appear to present fractionally significant difficulties to the utility of X-ray surveys for finding AGNs throughout the universe (see Section 1$)$. The majority ( $\gtrsim 80 \%$ ) of BAL quasars are also X-ray weak owing to intrinsic X-ray absorption (e.g., Gibson et al. 2009b). Considering the fraction of BAL quasars $(\approx 15 \%-20 \%$; e.g., Gibson et al. 2009b) found in SDSS quasar samples, X-ray weak BAL quasars outnumber X-ray weak PHL 1811 analogs by a factor of $\gtrsim 10$.

\subsection{Intrinsic X-Ray Weakness versus X-Ray Absorption}

Our radio-quiet PHL 1811 analogs could be X-ray weak either because they are intrinsically X-ray weak, perhaps due to quenching of the ADC (see Section 1), or because they are $\mathrm{X}$-ray absorbed. In this section, we will discuss the strengths and weaknesses of each of these hypotheses.

There are three indirect arguments supporting the intrinsic $\mathrm{X}$-ray weakness scenario. First, as discussed in Section 2.1, we have selected against objects with detectable broad $\mathrm{C}$ IV absorption (or any other broad absorption lines, including Si IV and MgII), since most heavily X-ray absorbed type 1 quasars show broad C IV absorption (e.g., Brandt et al. 2000; Gibson et al. 2008a; Page et al. 2010). If we assume the PHL 1811 analogs have underlying X-ray spectra with $\Gamma=2$, the level of intrinsic neutral absorption would need to be $N_{H} \approx 5 \times 10^{23} \mathrm{~cm}^{-2}\left(\gtrsim 2 \times 10^{23} \mathrm{~cm}^{-2}\right.$ to $\left.\gtrsim 8 \times 10^{23} \mathrm{~cm}^{-2}\right)$ at $z \approx 2.2$ to generate the observed $\mathrm{X}$-ray weakness factor of 13 (and the range of $>4.76$ to $\geqslant 34.5$ ). Three of our eight radio-quiet PHL 1811 analogs (J0848+5408, J1219+1244, and J1230+2049) have narrow associated C IV absorption lines (within $\pm 5000 \mathrm{~km} \mathrm{~s}^{-1}$ of the quasar redshift). Quasars with associated NALs generally have similar X-ray properties to unabsorbed quasars and are not heavily X-ray absorbed (e.g., Misawa et al. 2008; Chartas et al. 2009). The intrinsic X-ray absorption for quasars with associated NAL systems is typically $N_{H} \lesssim 10^{22} \mathrm{~cm}^{-2}$, much lower than the level required for our radio-quiet PHL 1811 analogs. The frequency of associated NAL systems in our sample $(3 / 8=38 \%)$ is consistent with that of typical quasars (see Section 3.2.1 of Ganguly \& Brotherton 2008). For the total number of NAL systems (both associated and intervening), the work of Nestor et al. (2008) predicts that between Ly $\alpha$ and $\mathrm{C}_{\mathrm{IV}}$ in the spectra of our eight radio-quiet PHL 1811 analogs we should find $16 \mathrm{C}$ IV absorbers with FWHM $<600 \mathrm{~km} \mathrm{~s}^{-1}$ and $W_{r}(\lambda 1548) \leqslant-0.3 \AA$. In fact, we find 10 . Within the limitations of small-number statistics, there is nothing unusual about the total number of NAL systems in our PHL 1811 analogs. One source, J1219+1244, has a C IV absorber with a total velocity width of $1100 \mathrm{~km} \mathrm{~s}^{-1}$, just above the $1000 \mathrm{~km} \mathrm{~s}^{-1}$ threshold to be termed a miniBAL quasar. Mini-BAL quasars are also generally not strongly X-ray absorbed (e.g., Gibson et al. 2009a; Wu et al. 2010a). The intrinsic X-ray absorption of mini-BAL quasars is also typically at the level of $N_{H} \lesssim 10^{22} \mathrm{~cm}^{-2}$ (Wu et al. 2010a). While it is perhaps possible that a type 1 quasar could be strongly X-ray absorbed without showing notable UV absorption lines (see below), the available empirical evidence indicates that our removal of quasars with BALs should eliminate the vast majority of strongly X-ray absorbed quasars.

Second, the quasars in our sample show the blue UV/optical continua typical of type 1 quasar spectra. Our sample was chosen to avoid objects with detectable spectral curvature indicative of strong dust reddening. Nonetheless, the somewhat redder than average colors of our sample (see Section 2.4) suggest that we cannot rule out an average dust reddening of up to $E(B-V) \approx 0.05$ magnitudes. For an SMC dust-to-gas ratio (Bouchet et al. 1985), such reddening would imply a column density of $N_{H} \approx 2.2 \times 10^{21} \mathrm{~cm}^{-2}$. Again, this column density is $\gtrsim 100$ times too small to explain the X-ray weakness of our radio-quiet PHL 1811 analogs. Note, however, that this is not a strong argument against X-ray absorption, since many BAL quasars (which usually have heavy X-ray absorption) also have blue UV/optical continua (e.g., Trump et al. 2006).

Third, the unusual UV emission-line properties of PHL 1811 can be explained naturally by an intrinsically X-ray weak SED (Leighly et al. 2007a). Such a continuum lacks adequate photons to create highly ionized ions, which results in weak highionization lines (e.g., C IV, Si IV). The temperature of the gas is too low to excite semi-forbidden lines (e.g., C III]). The strong Fe II and Fe III emission can also be qualitatively explained by the PHL 1811-like SED (Leighly et al. 2007a). Since our PHL 1811 analogs have the same unusual UV emission-line properties, Occam's razor would suggest their SEDs are also probably intrinsically X-ray weak.

In spite of the indirect arguments above, it is notable that the average X-ray spectrum for our radio-quiet PHL 1811 analogs appears to be harder than for typical radio-quiet quasars and for PHL 1811 itself (see Section 4.2), which hints at the presence of heavy X-ray absorption. ${ }^{18}$ It is possible that X-ray absorption could also lead to the X-ray weak SEDs required to create the unusual UV emission lines of our PHL 1811 analogs. However, if heavy X-ray absorption is responsible, it must lie closer to the SMBH than the BELR does, so that this emission-line region "sees" a soft ionizing SED and produces a PHL 1811-like UV emission-line spectrum. It must also have a remarkable lack of accompanying C IV, Si IV, or Mg II BALs (or even mini-BALs in all but one case). These requirements might be achieved by a line of sight that passes through "shielding gas" but not a typical UV absorbing wind (e.g., Gallagher et al. 2005). The shielding gas, which resides interior to the UV absorbing wind, is sufficiently highly ionized that it is transparent to UV photons but absorbs soft X-ray photons, preventing overionization of the wind (e.g., Murray et al. 1995). To explain PHL 1811 analogs, the shielding gas could have an atypically larger covering factor of the $\mathrm{X}$-ray continuum source that prevents most of the X-rays from reaching the BELR; we return to this idea in Section 4.6. The shielding gas might betray its presence via very highly ionized absorption, either in the UV or in X-rays. For example, Telfer et al. (1998) report that the X-ray weak quasar SBS $1542+541$

\footnotetext{
18 The harder average spectrum for these X-ray weak objects could also perhaps be explained if their X-ray spectra are reflection-dominated, similar to what may apply for PG 0844+349 in an X-ray weak state (Gallo et al. 2011).
} 
Table 7

Correlation Probabilities from Kendall's $\tau$ Tests and Spearman Rank-order Analyses

\begin{tabular}{|c|c|c|c|c|c|c|c|c|c|c|c|c|}
\hline \multirow[t]{3}{*}{ Correlation with $\Delta \alpha_{\mathrm{ox}}$} & \multicolumn{4}{|c|}{$\mathrm{RQQ}^{\mathrm{a}}(N=8)^{\mathrm{d}}$} & \multicolumn{4}{|c|}{$\mathrm{RQQ}+\mathrm{RIQ}^{\mathrm{b}}(N=10)^{\mathrm{d}}$} & \multicolumn{4}{|c|}{ RQQ+G08a $\mathrm{a}^{\mathrm{c}}+\mathrm{PHL} 1811(N=141)^{\mathrm{d}}$} \\
\hline & \multicolumn{2}{|c|}{ Kendall } & \multicolumn{2}{|c|}{ Spearman } & \multicolumn{2}{|c|}{ Kendall } & \multicolumn{2}{|c|}{ Spearman } & \multicolumn{2}{|c|}{ Kendall } & \multicolumn{2}{|c|}{ Spearman } \\
\hline & $\tau$ & $1-P_{\tau}$ & $r_{S}$ & $1-P_{S}$ & $\tau$ & $1-P_{\tau}$ & $r_{S}$ & $1-P_{S}$ & $\tau$ & $1-P_{\tau}$ & $r_{S}$ & $1-P_{S}$ \\
\hline C IV blueshift & 0.36 & $28.2 \%$ & 0.31 & $58.9 \%$ & 1.95 & $94.9 \%$ & 0.69 & $96.1 \%$ & 4.77 & $>99.99 \%$ & 0.39 & $>99.99 \%$ \\
\hline C IV FWHM & 1.10 & $72.7 \%$ & -0.19 & $38.7 \%$ & 0.98 & $67.2 \%$ & -0.28 & $60.6 \%$ & $\ldots$ & $\ldots$ & $\ldots$ & $\ldots$ \\
\hline C IV $\sigma_{\text {line }}$ & 1.10 & $72.7 \%$ & -0.56 & $85.8 \%$ & 0.73 & $53.7 \%$ & -0.41 & $77.5 \%$ & $\ldots$ & $\ldots$ & $\ldots$ & $\ldots$ \\
\hline C IV FWHM/ $\sigma_{\text {line }}$ & 0.36 & $28.2 \%$ & 0.31 & $58.9 \%$ & 0.00 & $0.0 \%$ & 0.11 & $25.0 \%$ & $\ldots$ & $\ldots$ & $\ldots$ & $\ldots$ \\
\hline$W_{r}(\mathrm{C}$ IV $)$ & 0.36 & $28.2 \%$ & -0.38 & $68.5 \%$ & 0.73 & $53.5 \%$ & -0.39 & $75.7 \%$ & 6.04 & $>99.99 \%$ & 0.49 & $>99.99 \%$ \\
\hline$W_{r}(\mathrm{Si} I \mathrm{~V})$ & 1.81 & $92.9 \%$ & 0.66 & $91.7 \%$ & 0.24 & $19.3 \%$ & 0.26 & $56.8 \%$ & $\ldots$ & $\ldots$ & $\ldots$ & $\ldots$ \\
\hline$W_{r}(\lambda 1900 \AA)$ & 0.36 & $28.2 \%$ & 0.10 & $21.6 \%$ & 0.00 & $0.0 \%$ & -0.00 & $1.7 \%$ & 1.20 & $76.88 \%$ & 0.11 & $80.32 \%$ \\
\hline$W_{r}(\mathrm{Fe}$ III $)$ & 1.10 & $72.7 \%$ & -0.61 & $89.2 \%$ & 0.00 & $0.0 \%$ & -0.20 & $44.9 \%$ & $\ldots$ & $\ldots$ & $\ldots$ & $\ldots$ \\
\hline
\end{tabular}

Notes.

a Radio-quiet PHL 1811 analogs in our sample.

${ }^{b}$ Radio-quiet and radio-intermediate PHL 1811 analogs in our sample.

${ }^{c}$ Radio-quiet, non-BAL quasars in Sample B of Gibson et al. (2008a).

d $N$ is the sample size. The Kendall's $\tau$ test is preferred when $N \leqslant 30$, otherwise the Spearman rank-order analysis is preferred.

has BAL troughs much stronger in $\mathrm{O}$ VI and $\mathrm{Ne}$ VIII than in $\mathrm{N} \mathrm{v}$ or C IV (see their Figure 5), and O VI and Ne VIII have been seen in narrower absorption systems as well (e.g., Scott et al. 2004).

In fact, PHL 1811 has a narrow O VI absorber at $z=0.192186$ (consistent with the Balmer-line redshift of PHL 1811 in Leighly et al. 2007a) in which the O vi column density is $\sim 100$ times the H I column density (Tripp et al. 2008). Our inspection of the HST Space Telescope Imaging Spectrograph (STIS) spectrum of PHL 1811 also reveals $\mathrm{N} v$ absorption at the same redshift, with $W_{r}$ an order of magnitude smaller than that of $\mathrm{O} \mathrm{vI}$; C IV absorption is not seen in a lower-resolution STIS spectrum. Associated $\mathrm{O}$ VI absorption is not rare, being seen in about 23\% (Frank et al. 2010) to 63\% (Tripp et al. 2008) of quasars. However, the $z=0.192186$ absorber in PHL 1811 is unusual, having the highest $\mathrm{O}$ vi column density and the highest $\mathrm{O}$ VI/ HI ratio among the 14 associated O VI absorbers in Tripp et al. (2008). If this unusual O VI absorber is connected with the unusual X-ray and UV properties of PHL 1811, future spectroscopy should reveal unusual associated O VI absorption in our PHL 1811 analogs as well.

In summary, the currently available data are unable to discriminate rigorously between the intrinsic X-ray weakness and $\mathrm{X}$-ray absorption scenarios. Occam's razor might initially suggest favoring the hypothesis that our PHL 1811 analogs are intrinsically X-ray weak like PHL 1811 itself appears to be. However, the suggestion of heavy X-ray absorption from the likely hard X-ray spectra definitely needs further investigation. If our objects are indeed established to be heavily X-ray absorbed, then Occam's razor might alternatively be used to motivate reconsideration of the evidence that PHL 1811 is intrinsically X-ray weak. The evidence for intrinsic X-ray weakness presented by Leighly et al. (2007b) appears strong (e.g., steep X-ray spectrum with no apparent photoelectric absorption cutoff, X-ray variability). Nevertheless, absorption effects in local AGNs are sometimes observed to be exceedingly complex, and perhaps some unusual absorbers or special absorption geometry for this unusual quasar might yet be consistent with the observed X-ray properties.

\subsection{Correlations between X-Ray Weakness and UV Emission-line Properties}

We have quantitatively searched for relations between relative $\mathrm{X}$-ray weakness and the unusual UV emission-line properties.
Correlation analyses were performed between $\Delta \alpha_{\mathrm{ox}}$ and the UV emission-line parameters listed in Table 3, using both Kendall's $\tau$ test and Spearman's rank-order analysis in the ASURV package. Kendall's $\tau$ test is preferred over Spearman's rankorder analysis in small-sample cases, where the sample size is $N \leqslant 30 .{ }^{19}$ The results are presented in Table 7 . We found only a marginal correlation (94.9\% correlation probability) between $\Delta \alpha_{\text {ox }}$ and the blueshift of the C IV line when including both radio-quiet and radio-intermediate PHL 1811 analogs in our sample. The effective significance of this correlation is further weakened by the number of trials, since we have tested for multiple correlations in Table 7. No other correlations were found; this is likely due to the small sample size and the limited range of parameter space spanned by the PHL 1811 analogs alone. If we use the redshift values of Hewett \& Wild (2010) for our PHL 1811 analogs, the correlation between $\Delta \alpha_{\text {ox }}$ and the C IV blueshift remains insignificant ( $91.2 \%$ correlation probability).

Figure 6 shows $\Delta \alpha_{\text {ox }}$ plotted against the UV emission-line parameters of $W_{r}(\mathrm{C}$ IV $), W_{r}(\lambda 1900 \AA)$, and C IV blueshift for radio-quiet PHL 1811 analogs, PHL 1811 itself, and the radioquiet, non-BAL quasars in Sample B of Gibson et al. (2008a). The addition of the Sample B quasars and PHL 1811 increases the sample size to $N=148$ and broadens the parameter space of line measurements. The values of $W_{r}(\mathrm{C}$ IV $)$ and C IV blueshift for the quasars in Sample B are taken from Shen et al. (2011); we modified the C IV blueshift values for the Sample B quasars to account for the improved redshift measurements of Hewett \& Wild (2010). The values of $W_{r}(\lambda 1900 \AA)$ are taken from Gibson et al. (2008a). PHL 1092 (see Section 1) also has PHL 1811-like UV emission-line properties and shows dramatic $\mathrm{X}$-ray variability. ${ }^{20}$ We include this source in Figures 6 and 7, but not in the correlation analyses.

A significant correlation between $W_{r}(\mathrm{C}$ IV $)$ and $\Delta \alpha_{\text {ox }}$ was reported by Gibson et al. (2008a) for their sample. After adding our radio-quiet PHL 1811 analogs and PHL 1811, the correlation

\footnotetext{
${ }^{19}$ See Appendix A3.3 of the ASURV manual at http://astrostatistics.psu.edu/statcodes/asurv.

20 We have inspected the available rest-frame UV spectra of PHL 1092 (Bergeron \& Kunth 1980; Leighly et al. 2007a), covering Ly $\alpha$, Si IV, C IV, C III], Fe III UV48, and Mg II, and measured its UV emission-line parameters as listed in Table 3. We do not have spectral coverage of its UV Fe II emission. PHL 1092 likely would have been selected as a PHL 1811 analog by our criteria in Section 2, if it were in our redshift selection range of $2.125 \leqslant z \leqslant 2.385$ and were sufficiently bright.
} 

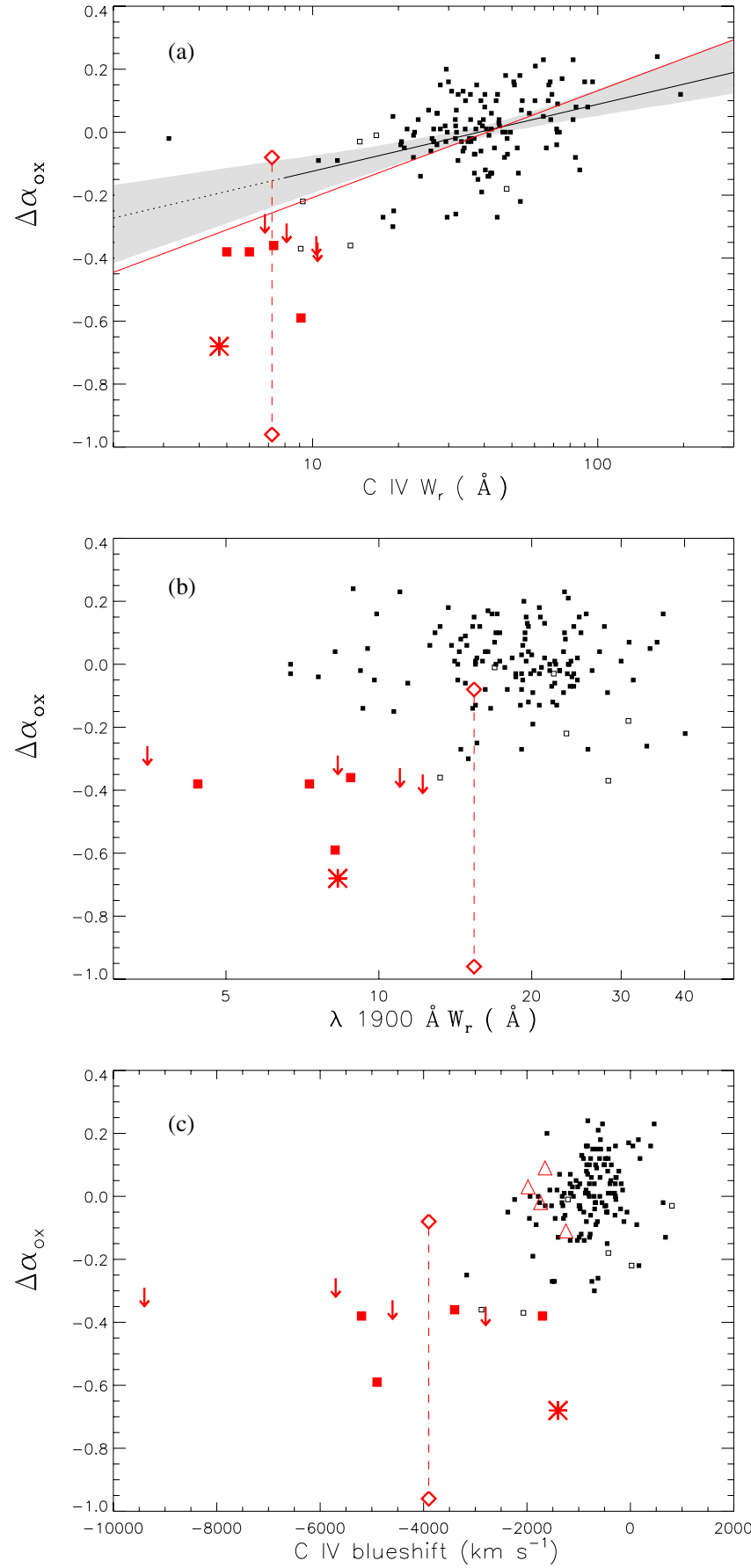

Figure 6. Values of $\Delta \alpha_{\text {ox }}$ plotted against the UV emission-line parameters of $W_{r}\left(\mathrm{C}\right.$ IV) (a), $W_{r}(\lambda 1900 \AA)$ (b), and C IV blueshift (c). The black filled squares are radio-quiet, non-BAL quasars in Sample B of Gibson et al. (2008a). The black open squares represent six sample B quasars that have possible BAL or mini-BAL features based on visual inspection (see footnote "16"). All Sample B quasars shown here are X-ray detected. The red filled squares and downward arrows represent the radio-quiet PHL 1811 analogs detected and undetected in X-rays, respectively. The red asterisk represents PHL 1811. The red open diamonds represent the highly X-ray variable source PHL 1092 in two XMMNewton observations in 2003 (with greater $\Delta \alpha_{\text {ox }}$ value) and 2008. The bestfit linear correlation for the Sample B quasars is shown by the black line in panel (a); the solid part represents the range where Gibson et al. (2008a) had statistically constraining data, while the dotted part is an extrapolation of the solid line. The shaded area shows the $95 \%$ confidence uncertainty range for this linear correlation determined with a nonparametric bootstrap method. The red solid line shows the best-fit correlation for a sample combining our radio-quiet PHL 1811 analogs, PHL 1811, and Sample B quasars. The red open triangles in panel (c) show the four quasars from Gallagher et al. (2005) having "large" C Iv blueshift values.

(A color version of this figure is available in the online journal.) becomes more significant (see Table 7 and the red solid line in Figure 6(a)). However, all of the radio-quiet PHL 1811 analogs and PHL 1811 itself lie well below the best-fit linear correlation (in log space) in Gibson et al. (2008a) between $W_{r}(\mathrm{C}$ IV $)$ and $\Delta \alpha_{\text {ox }}$. This remains true even if we consider the $95 \%$ confidence uncertainty range shown in Figure 6(a), obtained using a nonparametric bootstrap method (Efron 1979). This deviation from the correlation found for typical radio-quiet quasars may indicate that the radio-quiet PHL 1811 analogs arise from a distinct population of typical radio-quiet quasars. There is no significant correlation between $W_{r}(\lambda 1900 \AA)$ and $\Delta \alpha_{\text {ox }}$ (see Table 7 and Figure 6(b)).

The CIV emission lines of quasars are generally known to have systematic blueshifts (e.g., Gaskell 1982; Richards et al. 2011). We performed Spearman rank-order analysis and found a correlation between C IV blueshift and $\Delta \alpha_{\text {ox }}$ for radio-quiet PHL 1811 analogs, PHL 1811, and the Sample B quasars. However, this correlation shows substantial scatter (see Figure 6(c)). Although the correlation probability $(>99.99 \%)$ is formally high, the correlation coefficient $\left(r_{S}=0.39\right)$ shows that this correlation is weaker than that between $W_{r}(\mathrm{C}$ IV $)$ and $\Delta \alpha_{\text {ox }}$ $\left(r_{S}=0.49\right)$. Furthermore, it is difficult to determine if there is truly a single correlation or instead two populations artificially producing an apparent correlation.

The red triangles in Figure 6(c) show the four radioquiet, non-BAL quasars from Gallagher et al. (2005) which have "large" C IV blueshifts compared to most typical radioquiet, non-BAL quasars. Their X-ray brightnesses appear to be normal. However, the magnitudes of their C IV blueshifts are $\leqslant 2000 \mathrm{~km} \mathrm{~s}^{-1}$, while all but one of the radio-quiet PHL 1811 analogs in our sample have C IV blueshift magnitudes $>2000 \mathrm{~km} \mathrm{~s}^{-1}$. The four sources from Gallagher et al. (2005) are more similar to the typical radio-quiet, non-BAL quasars. Gallagher et al. (2005) also suggested evidence for intrinsic absorption (at the level of $N_{H} \approx 10^{22} \mathrm{~cm}^{-2}$ ) for their "large" $\mathrm{C}$ IV blueshift objects via joint X-ray spectral analyses. In order to search for any possible trend between intrinsic absorption and the C IV blueshift, we performed a band-ratio analysis for all the Sample B quasars from Gibson et al. (2008a). A harder $\mathrm{X}$-ray spectrum (with smaller effective photon index) could indicate the presence of intrinsic absorption. However, no correlation was found between the effective photon indices and C IV blueshifts.

In Figure 7 , the $\mathrm{C}$ IV blueshift is plotted against $W_{r}(\mathrm{C}$ IV $)$ for our radio-quiet PHL 1811 analogs, PHL 1811, and the Sample B quasars in Gibson et al. (2008a). The color for each source shows its $\Delta \alpha_{\text {ox }}$ value, indicating its relative $\mathrm{X}$-ray brightness. The gray dots represent the 13,582 radioquiet Sample A quasars from Richards et al. (2011) which do not generally have constraining information on their $\mathrm{X}$-ray properties. Motivated by much past work, Richards et al. (2011) described a BELR model with both "disk" and "wind" components. The $\mathrm{C}$ IV blueshift and $W_{r}(\mathrm{C}$ IV $)$ show the tradeoff between these two components, which ultimately depends upon the shape of the ionizing continuum. The claimed interpretation is that quasar BELRs change from disk-dominated (with a more ionizing SED) to wind-dominated (with a less ionizing SED) as a quasar's location moves from the upper right corner to the lower left corner of Figure 7. The relative X-ray brightness becomes moderately weaker for the Sample B quasars in Gibson et al. (2008a) following this trend. Our radio-quiet PHL 1811 analogs show extreme behavior in the $\Delta \alpha_{\text {ox }}-\mathrm{C}$ IV blueshift $-W_{r}(\mathrm{C}$ IV $)$ parameter space; note they are essentially 


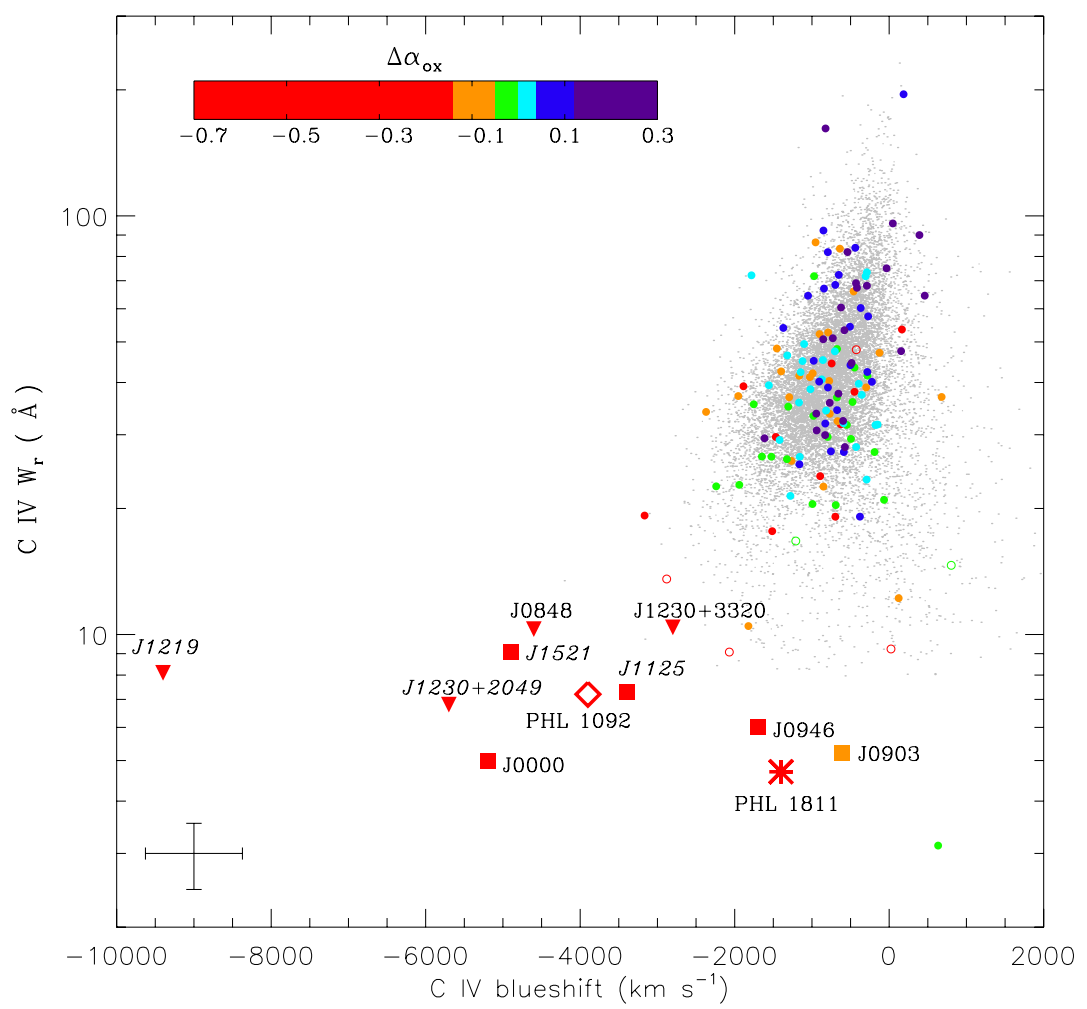

Figure 7. C IV blueshift plotted against $W_{r}(\mathrm{C}$ IV $)$ for our radio-quiet PHL 1811 analogs (filled squares for X-ray detected sources, filled upside-down triangles for X-ray undetected sources), PHL 1811 (asterisk), and radio-quiet, non-BAL quasars in Sample B of Gibson et al. (2008a, filled circles). The open circles represent six sample B quasars that have possible BAL or mini-BAL features based on visual inspection (see footnote " 16 "). These sources are color-coded according to their $\Delta \alpha_{\text {ox }}$ values (six color bins are used, each chosen to contain an approximately equal number of objects). The color bar shows the $\Delta \alpha_{\text {ox }}$ range for each color. Source names for PHL 1811 analogs are labeled in the format of "Jhhmm" for brevity, except for J1230+3320 and J1230+2049. The sources with italic names are the PHL 1811 analogs for which we adopt different redshift values other than those from the SDSS CAS. J0903+0708 is also shown using the line measurements from its HET spectrum. The red open diamond represents PHL 1092. The red color shows its X-ray weakness from the latest XMM-Newton observation (see Miniutti et al. 2009). The median errors for $\mathrm{C}$ IV blueshifts and $W_{r}(\mathrm{C}$ IV $)$ of our PHL 1811 analogs are shown in the lower left corner of the figure (the blueshift error includes contributions from both redshift measurement and C IV wavelength measurement). The gray dots show the 13,582 radio-quiet quasars in Sample A of Richards et al. (2011, see their Figure 7).

(A color version of this figure is available in the online journal.)

disjoint from the main quasar population. This suggests they may have extreme wind-dominated BELRs.

\subsection{The Relation between PHL 1811 Analogs and Weak-line Quasars}

The SDSS has discovered $\sim 80$ high-redshift $(z>2.2)$ quasars with extremely weak or undetectable UV emission lines (WLQs; e.g., Diamond-Stanic et al. 2009); similar objects also likely exist at lower redshifts (e.g., Plotkin et al. 2010a). In this section, we discuss the observational relation between our PHL 1811 analogs and WLQs more generally. WLQs are defined as quasars with $W_{r}(\mathrm{Ly} \alpha+\mathrm{Nv})<15.4 \AA$, measured between $1160 \AA$ and $1290 \AA$ in the rest frame (DiamondStanic et al. 2009). Measurements of $\operatorname{Ly} \alpha W_{r}$ are difficult with our wavelength coverage, but many of our PHL 1811 analogs likely meet the WLQ definition; for example, J0946+2744 was identified as a WLQ by Shemmer et al. (2009).

There has been some past speculation about a possible connection between PHL 1811 itself and WLQs (e.g., Leighly et al. 2007a; Shemmer et al. 2009), but we here have the advantage of having a carefully selected sample of PHL 1811 analogs with established basic X-ray properties. Figure 8 compares the median composite spectrum of radio-quiet PHL 1811 analogs with the mean spectra of WLQs from Shemmer et al. (2009) and
Diamond-Stanic et al. (2009). ${ }^{21}$ PHL 1811 analogs and WLQs both have weak Ly $\alpha$, Si IV, and C IV emission lines. PHL 1811 analogs have prominent UV Fe II and Fe III emission, while most WLQ samples have been selected at high redshift and generally lacked spectral coverage of UV Fe emission. However, inspection of the lower-redshift WLQ samples of Plotkin et al. (2010a, Plotkin et al. 2010b) shows that their UV Fe emission can be just as prominent relative to the broad lines as it is in PHL 1811 analogs. Furthermore, the two WLQs studied by Shemmer et al. (2010) both have prominent optical Fe II, similar to PHL 1811 itself (Leighly et al. 2007a).

When defining our sample of PHL 1811 analogs (see Section 2.1), we first selected 54 objects with weak highionization lines, 32 of which can be considered non-BAL WLQs. Twenty-nine of the 32 non-BAL WLQs are radio quiet. Eight of these 29 sources having strong UV Fe emission and C IV blueshifts were retained as our radio-quiet PHL 1811 analogs. At least from an observational point of view, PHL 1811 analogs thus appear to be a subset $(\simeq 30 \%)$ of WLQs. Our radioquiet PHL 1811 analogs are X-ray weak compared to typical

\footnotetext{
21 Because our PHL 1811 analogs all lie in a similar part of the $W_{r}(\mathrm{C}$ IV $)$ versus C IV blueshift parameter space, their composite spectrum should be physically meaningful at least to first order (cf. Section 5 of Richards et al. 2011).
} 


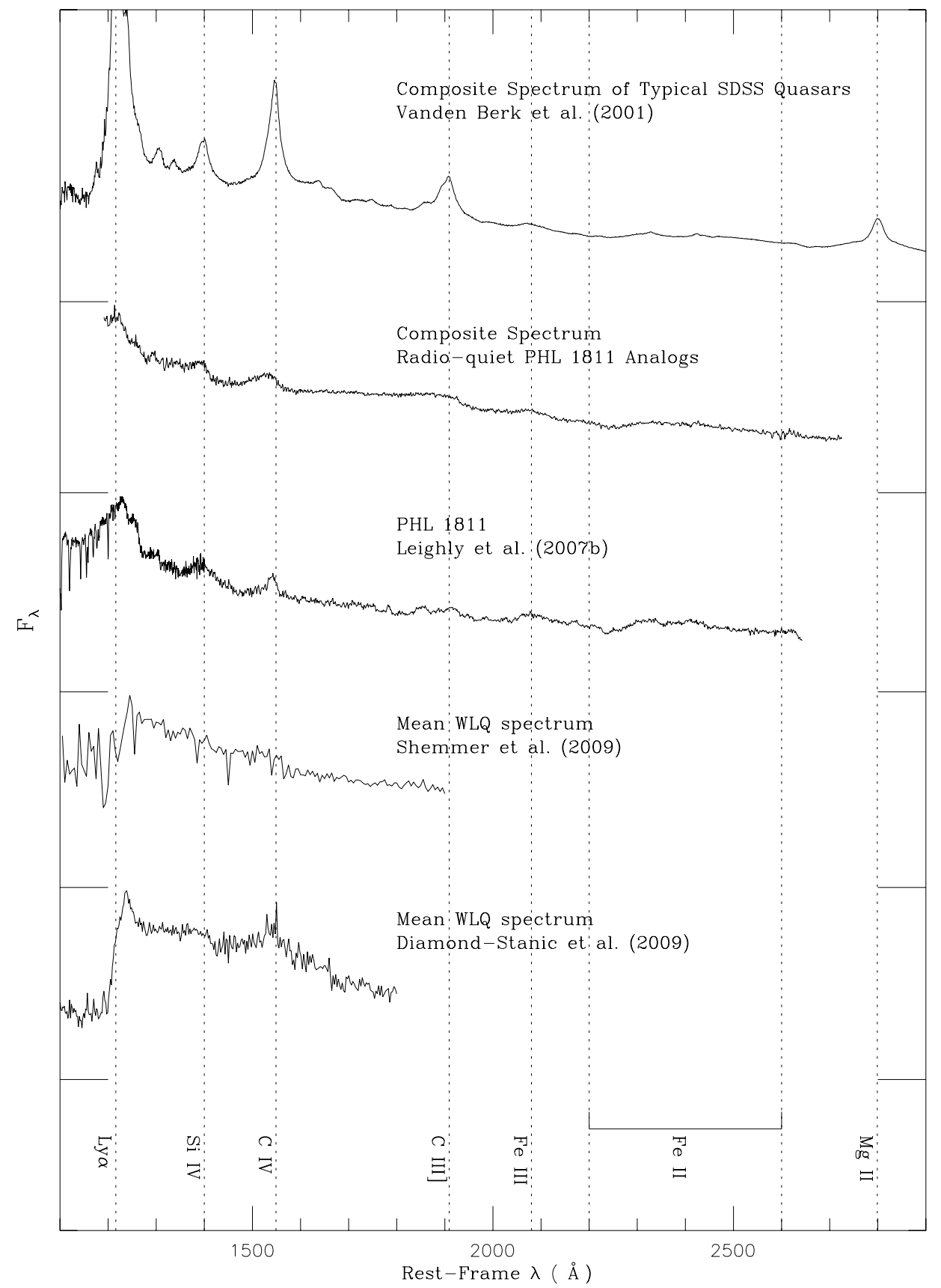

Figure 8. Comparison of the median composite spectrum of radio-quiet PHL 1811 analogs with the mean spectra of WLQs from Shemmer et al. (2009), and Diamond-Stanic et al. (2009). The $y$-coordinates are the flux density $\left(F_{\lambda}\right)$ in arbitrary linear units. The tick marks on the $y$-axis show the zero flux density level for each normalized spectrum. The emission lines are labeled by the dotted vertical lines. Also shown are the median composite spectrum of typical SDSS quasars from Vanden Berk et al. (2001) and the spectrum of PHL 1811 from HST (Leighly et al. 2007a).

quasars, while WLQs are generally not X-ray weak as a population (Shemmer et al. 2009). However, there are a few known X-ray weak WLQs (e.g., J1302+0030, J1421+3433, and perhaps J1335+3533 and J1532-0039 in Shemmer et al. 2006, 2009) that may be PHL 1811 analogs. We do not have reliable measurements of the CIV blueshifts for these sources because of either the wavelength coverage or the quality of their SDSS spectra. Near-infrared spectroscopy for many WLQs (e.g., Shemmer et al. 2010) is required to assess if the X-ray weak WLQs have notably strong UV Fe II and Fe III emission.

While the observational relation between PHL 1811 analogs and WLQs appears fairly simple, as described above, any physical relation between these two classes may be much more complex. The X-ray weakness and distinctive emission lines of PHL 1811 analogs may ultimately be due to some extreme physical parameter (or parameters), such as accretion rate (see Section 1). In contrast, at least some WLQs appear simply to have anemic broad line regions (Shemmer et al. 2010), and some may be in a specific evolutionary stage where the quasar activity has only recently begun (Hryniewicz et al. 2010).

However, there is another scenario that might unify PHL 1811 analogs and most WLQs, which we illustrate in Figure 9. As mentioned in Section 4.4, suppose there is a subset of quasars in which the shielding gas covers all or most of the BELR (which likely consists of "clouds"), but little more than the BELR. A sufficient column of such gas could absorb 
Normal Quasars

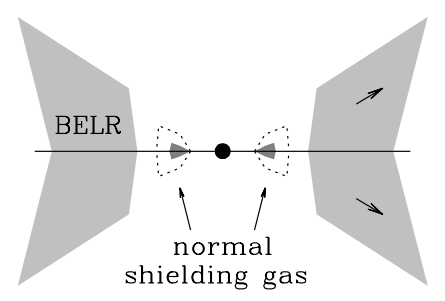

Unified WLQs / PHL 1811 Analogs

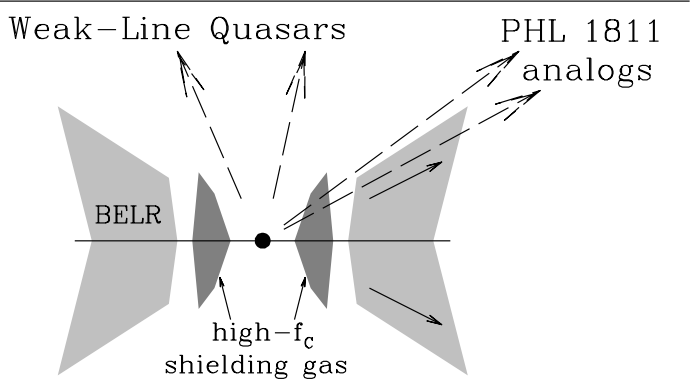

Figure 9. Schematic illustration of the WLQ/PHL 1811 analog unification hypothesis. Each panel is a side view of an accretion disk around a black hole, showing the X-ray and UV continuum sources (central black dot), shielding gas (gray), and BELR (light gray). The BELR is shown as shaded regions for convenience, but likely consists of "clouds." Normally (top panel), the shielding gas only covers part of the BELR, resulting in moderate emission-line blueshifts at best (short arrows in the BELR). The dotted outline illustrates the fact that normal quasars are expected to have a range of shielding gas covering factors $\left(f_{\mathrm{C}}\right)$ and columns (and thus a range of wind strengths). When the shielding gas has a BELR covering factor of $f_{\mathrm{C}} \gtrsim 80 \%$ (bottom panel), a strong wind is generated (long arrows in the BELR). When such a quasar is viewed through the shielding gas and thus close to the wind direction, a PHL 1811 analog is seen (X-ray absorbed, high blueshift). When such a quasar is viewed away from the wind direction, a weak-line quasar is seen (X-ray normal, low blueshift).

X-ray and other ionizing photons before they reach the BELR, resulting in weak broad-line emission and wind acceleration without overionization. (In normal quasars with strong broadline emission, a range of shielding gas covering factors and columns are likely to exist, but the majority of the BELR gas cannot be shielded by such high columns and must be exposed to the ionizing continuum.) When such quasars are observed through the shielding gas, a PHL 1811 analog can be seen; when they are observed from other directions (where shielding gas is unlikely to be seen), an X-ray normal WLQ can be seen. A PHL 1811 analog fraction of 30\% among WLQs is consistent with the estimated BELR covering fraction (e.g., Maiolino et al. 2001). The low C IV $W_{r}$ values in PHL 1811 analogs require that $\gtrsim 80 \% \pm 20 \%$ of the BELR be covered by shielding gas. ${ }^{22}$ As coverage of randomly distributed BELR clouds by shielding gas with the same covering factor would be physically implausible, this scenario requires the BELR in PHL 1811 analogs and WLQs to have a non-random geometrical distribution (see, e.g., Section 4.3 of Risaliti et al. 2011 and references therein). The large C IV blueshifts of PHL 1811 analogs could be produced in lines of sight into an accelerating wind, for example, from a rotating, disk-like BELR (Murray et al. 1995).

22 We have illustrated this using shielding gas of variable $f_{\mathrm{C}}$ in Figure 9. Alternatively, as depicted in Figure 15 of Leighly (2004), an X-ray source of varying height above the disk could result in varying fractions of the BELR being illuminated by X-rays (see also Miniutti \& Fabian 2004).
This simple unification scenario makes several predictions. The O VI absorption in PHL 1811 (see Section 4.4) is evidence for the existence of highly ionized UV-absorbing gas along our sightline to its central engine; PHL 1811 analogs might have an excess of such absorption, but WLQs should not. Broad emission line $W_{r}$ values in WLQs and PHL 1811 analogs should be comparable, consistent with the limited data available to date (e.g., the Appendix and Shemmer et al. 2010). Narrow-line emission could be seen in WLQs and PHL 1811 analogs in this model, but is unlikely given the observed anti-correlation (Boroson \& Green 1992; Netzer et al. 2004) of [O III] strength with the $\mathrm{Fe}$ II $/ \mathrm{H} \beta$ ratio, ${ }^{23}$ which is large in PHL 1811 (Leighly et al. 2007a), J1521+5202 (see the Appendix), and at least two WLQs (Shemmer et al. 2010). Additional observations to test these predictions are needed.

\subsection{Radio-intermediate PHL 1811 Analogs}

The two radio-intermediate sources in our sample, $\mathrm{J} 1454+0324$ and $\mathrm{J} 1618+0704$, are X-ray bright with $\Delta \alpha_{\mathrm{ox}}=0.42$ and $\Delta \alpha_{\mathrm{ox}}=0.28$, respectively. Radio-intermediate sources probably have significant relativistic jets, which often have associated X-ray emission (e.g., Worrall et al. 1987; Miller et al. 2011). Figure 10 compares the X-ray "excesses" in these two PHL 1811 analogs (assessed with $\Delta \alpha_{\text {ox }}$ ) with those of typical radio-intermediate quasars from the primary sample of Miller et al. (2011). The $\Delta \alpha_{\text {ox }}$ values of our two radio-intermediate PHL 1811 analogs are relatively large compared to those of typical radio-intermediate quasars with similar radio loudness values ( $R=10-50)$, and also compared to the whole radiointermediate quasar sample ( $R=10-100$; also see Figure 7 of Miller et al. 2011). Note that the radio-loudness values in Miller et al. (2011) have been converted to our definition (they used $f_{2500 \AA}$ for the optical flux density).

We performed X-ray spectral analyses for the two radiointermediate PHL 1811 analogs, since both of them have sufficient counts for basic spectral fitting. The X-ray spectra were extracted with the PSEXTRACT routine in CIAO v4.2, using a $3^{\prime \prime}$ radius aperture centered on the X-ray position for each source. Background spectra were extracted using annular regions with inner radii of $6^{\prime \prime}$ and outer radii of $9^{\prime \prime}$. Both background regions are free of X-ray sources. Spectral fitting was executed using XSPEC v12.5.1 (Arnaud 1996). The X-ray spectra were grouped to have at least 20 (10) counts per bin for $\mathrm{J} 1454+0324$ (J1618+0704). We used a power-law model with a Galactic absorption component, in which the Galactic column density is fixed to the values from Table 5. We also tried another model similar to the first, but adding an intrinsic neutral absorption component. Table 8 shows the X-ray spectral fitting results. The quoted errors or the upper limits for the best-fit parameters are at $90 \%$ confidence for one parameter of interest $\left(\Delta \chi^{2}=2.71\right.$; Avni 1976). For both sources, we found no evidence for strong intrinsic neutral absorption; adding an intrinsic neutral absorption component did not improve the fit quality. The best-fit photon indices for both sources are consistent with those from band-ratio analysis (see the last column of Table 4 ).

$\mathrm{J} 1618+0704$, which has moderate radio emission $(R=35)$, has a relatively flat $X$-ray power-law continuum $\left(\Gamma=1.48_{-0.28}^{+0.29}\right)$ indicating that its X-ray flux probably has a substantial contribution from relativistic jets (e.g., Wilkes \& Elvis 1987;

${ }^{23} \mathrm{Fe} \mathrm{II} / \mathrm{H} \beta$ is defined as the ratio between $W_{r}(\mathrm{Fe}$ II $)$ in $4434-4684 \AA$ and $W_{r}(\mathrm{H} \beta)$. 


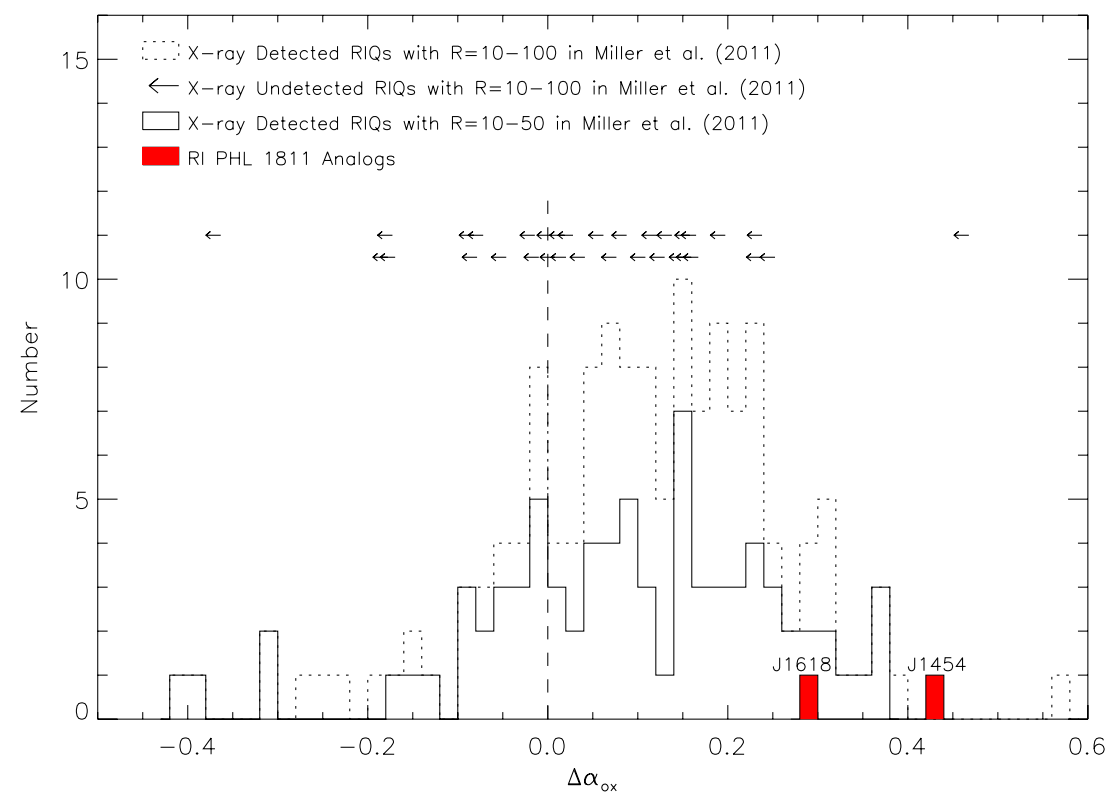

Figure 10. Comparison of the $\Delta \alpha_{\mathrm{ox}}$ value distributions for our radio-intermediate PHL 1811 analogs and typical radio-intermediate quasars in the primary sample of Miller et al. (2011). The red histogram represents our radio-intermediate PHL 1811 analogs, with their names labeled in the format of "Jhhmm" for brevity. The solid histogram shows the $\Delta \alpha_{\text {ox }}$ value distribution of X-ray detected typical radio-intermediate quasars with similar radio-loudness values $(R=10-50)$ to our PHL 1811 analogs, while the dotted histogram is for the whole sample of X-ray detected radio-intermediate quasars in Miller et al. (2011). The leftward arrows are for the X-ray undetected radio-intermediate quasars in Miller et al. (2011). The dashed vertical line shows $\Delta \alpha_{\mathrm{OX}}=0$.

(A color version of this figure is available in the online journal.)

Table 8

X-ray Spectral Analysis

\begin{tabular}{|c|c|c|c|c|c|}
\hline \multirow[t]{2}{*}{ Object Name (SDSS J) } & \multicolumn{2}{|c|}{$\begin{array}{c}\text { Power Law } \\
\text { with Galactic Absorption }\end{array}$} & \multicolumn{3}{|c|}{$\begin{array}{c}\text { Power Law } \\
\text { with Galactic and Intrinsic Absorption }\end{array}$} \\
\hline & $\Gamma$ & $\chi^{2} / v$ & $\Gamma$ & $N_{H}\left(10^{22} \mathrm{~cm}^{-2}\right)$ & $\chi^{2} / v$ \\
\hline $145453.53+032456.8$ & $2.13_{-0.17}^{+0.18}$ & $10.25 / 16$ & $2.13_{-0.16}^{+0.29}$ & $<1.36$ & $10.25 / 15$ \\
\hline $161801.71+070450.2$ & $1.48_{-0.28}^{+0.29}$ & $18.70 / 14$ & $1.48_{-0.27}^{+0.41}$ & $<1.98$ & $18.70 / 13$ \\
\hline
\end{tabular}

Page et al. 2005). The X-ray brightness and X-ray spectral shape of J1618+0704 suggest that the X-ray emission associated with jets has not been significantly diminished, even if the X-ray emission from the ADC has been absorbed or quenched as for the radio-quiet PHL 1811 analogs (i.e., the observed spectrum may be dominated by jet-linked emission).

The other radio-intermediate PHL 1811 analog, J1454+0324, has a steeper X-ray continuum $\left(\Gamma=2.13_{-0.17}^{+0.18}\right)$. The radioloudness value $(R=12.8)$ of this source is just above the upper limit for radio-quiet sources. However, J1454+0324 is brighter in X-rays than most typical radio-intermediate quasars with significantly higher $R$ values (see Figure 10). The notable $\mathrm{X}$-ray brightness and the steep X-ray continuum indicate that this source may have a different physical nature from that of $\mathrm{J} 1618+0704$. One possibility is that J1454+0324 may be similar to BL Lac objects. Although BL Lac objects are generally radioloud, it is possible that the BL Lac population has a small radio-faint tail (e.g., Plotkin et al. 2010a). In this scenario, J1454+0324 would have a relatively featureless UV spectrum because its emission lines have been somewhat diluted by a relativistically boosted UV continuum (though not enough to make it a bona fide BL Lac object), which allows it to pass our criteria for selecting PHL 1811 analogs. The $\alpha_{\text {ox }}$ value for $\mathrm{J} 1454+0324$ is consistent with those for the majority of BL Lac objects (e.g., Shemmer et al. 2009; Plotkin et al. 2010b) for which the X-ray emission is relativistically boosted. The $\mathrm{X}$-ray spectral slope of $\mathrm{J} 1454+0324$ is also consistent with those of BL Lac objects (e.g., Donato et al. 2005). However, there are several problems with a BL Lac-like interpretation. First, J1454+0324 has relatively strong observed Fe II and Fe III emission, which should also be diluted by a relativistically boosted continuum. Second, J1454+0324 has a blue UV/ optical continuum $\left(\alpha_{\nu}=-0.28\right)$, while typical BL Lac objects have red continua (e.g., Stein et al. 1976). Third, there is no significant variability (only $\approx 3 \%$ level) between the two SDSS spectroscopic epochs of J1454+0324. Future observations (e.g., polarization measurements) of J1454+0324 in the UV/optical band may further constrain its nature which presently remains uncertain.

\section{SUMMARY AND FUTURE STUDIES}

We report on the $\mathrm{X}$-ray properties of 10 quasars identified by the SDSS at high redshift $(z \approx 2.2)$. Seven of them have new Chandra observations, while three have archival Chandra or $X M M$-Newton data. This sample of 10 quasars (eight radioquiet, two radio-intermediate) was selected to have unusual UV emission-line properties similar to those of PHL 1811, a confirmed intrinsically X-ray weak quasar. Our main results are the following.

1. Four of the eight radio-quiet PHL 1811 analogs are detected in X-rays. The distribution of $\Delta \alpha_{0 x}$ values for radio-quiet PHL 1811 analogs is substantially different from that of typical radio-quiet, non-BAL quasars. All of the eight 
radio-quiet $\mathrm{PHL} 1811$ analogs, without exception, are anomalously $\mathrm{X}$-ray weak by a mean factor of $\approx 13$.

2. The fraction of radio-quiet PHL 1811 analogs in the total radio-quiet, non-BAL quasar population is estimated to be $\lesssim 1.2 \%$, and within the range of $0.3 \%-2.8 \%$ (at the $90 \%$ confidence level). The small fraction of PHL 1811 analogs does not present material difficulties to the utility of X-ray surveys for finding AGNs.

3. The currently available data are unable to discriminate rigorously between the intrinsic X-ray weakness and heavy X-ray absorption scenarios. The radio-quiet PHL 1811 analogs have blue UV/optical continua without detectable broad absorption lines or significant dust reddening, supporting the hypothesis that they are intrinsically X-ray weak like PHL 1811 itself. Their unusual UV emission-line properties can also be naturally explained by a model with an intrinsically X-ray weak SED. However, a stacking analysis of the radio-quiet PHL 1811 analogs shows evidence for a hard X-ray spectrum (with large error bars), which could be due to the presence of X-ray absorption. In this scenario, the X-ray absorption must occur on a scale smaller than the BELR and must cover most of the BELR. It is possible that very high-ionization (e.g., O vI) absorbers could be connected to the unusual X-ray and UV properties of PHL 1811 analogs and PHL 1811 itself.

4. Our sample of radio-quiet PHL 1811 analogs empirically supports the connection between soft SEDs (X-ray weak and UV/optical strong) and PHL 1811-like UV emission lines.

5. We have investigated correlations between relative $\mathrm{X}$-ray brightness and UV emission-line properties [e.g., $W_{r}(\mathrm{C}$ IV $)$ and $\mathrm{C}$ IV blueshift] for a sample combining our radio-quiet PHL 1811 analogs, PHL 1811, and typical type 1 quasars. A significant correlation is found between $W_{r}(\mathrm{C}$ IV $)$ and $\Delta \alpha_{\text {ox }}$. However, our radio-quiet PHL 1811 analogs show a notable deviation from the best-fit power-law correlation for typical type 1 quasars. The behavior of PHL 1811 analogs in the $\Delta \alpha_{\mathrm{ox}}-\mathrm{C}$ IV blueshift $-W_{r}(\mathrm{C}$ IV $)$ parameter space suggests that they may have extreme wind-dominated broad emission-line regions and/or may be a distinct population from typical radio-quiet quasars.

6. From an observational point of view, PHL 1811 analogs appear to be a subset $(\simeq 30 \%)$ of WLQs that are X-ray weak and have strong UV Fe emission and C IV blueshifts. The existence of a subset of quasars in which a large column of high-ionization shielding gas exists along all or most of the sightlines to the BELR, but along very few other sightlines, could potentially unify the PHL 1811 analogs and WLQs.

7. The two radio-intermediate PHL 1811 analogs are X-ray bright. One (J1618+0704) has an X-ray spectrum consistent with jet-dominated X-ray emission, and the other (J1454+0324) is more similar to BL Lac objects in terms of its X-ray brightness and spectral shape. However, for $\mathrm{J} 1454+0324$, the strong UV Fe emission, blue UV/optical continuum, and lack of variability do not support a BL Lac-like interpretation.

The observed connection between PHL 1811-like UV emission lines and soft SEDs appears to provide a practical and economical way to find X-ray weak quasars. X-ray observations of further targets selected using high-quality UV/ optical spectroscopy from large-area surveys, such as SDSS-III (Eisenstein et al. 2011), LAMOST (Su et al. 1998; Wu et al. 2010b), and BigBOSS (Schlegel et al. 2009), should identify many more PHL 1811 analogs that are X-ray weak.

Further X-ray studies of PHL 1811 analogs would be helpful to clarify their nature. Four of our eight radio-quiet PHL 1811 analogs are undetected in X-rays, leaving only upper limits on their X-ray fluxes. Deeper X-ray observations are required to convert the individual upper limits into detections and clarify the distribution of X-ray weakness for PHL 1811 analogs. A clear bimodality in the distribution of the relative X-ray brightness $\left(\Delta \alpha_{\mathrm{ox}}\right)$ for a sample combining typical type 1 quasars and PHL 1811 analogs would suggest that PHL 1811 analogs are indeed a distinct population of quasars; alternatively, the lack of such bimodality would suggest that PHL 1811 analogs simply represent one extreme of a continuum of quasar properties. Furthermore, our current sample of PHL 1811 analogs suffers from limited sample size largely because of the restricted redshift range used in target selection (see Figure 1). Selection over a broader range of redshift will allow us to investigate additional optically bright objects. An improved sample size will better characterize general X-ray properties and allow more reliable correlation tests with emission-line properties. X-ray spectroscopy should be able to test directly if PHL 1811 analogs are intrinsically X-ray weak; extensive X-ray observations provided strong evidence that the X-ray weakness of PHL 1811 was intrinsic rather than due to absorption (Leighly et al. 2007b). A stacking analysis suggests the average X-ray spectrum of radio-quiet PHL 1811 analogs is harder than those of typical quasars, which may indicate the presence of heavy X-ray absorption. Stacking analyses with deeper and/or more observations can test and extend this result with better photon statistics. If the X-rays are absorbed, we expect to see a photoelectric absorption cutoff in highquality X-ray spectra, which can rigorously distinguish the intrinsic X-ray weakness and the heavy X-ray absorption scenarios. X-ray spectral measurements of the photon index of the hard X-ray power law can also constrain $L / L_{\text {Edd }}$ values (e.g., Shemmer et al. 2008), providing insight as to whether high $L / L_{\text {Edd }}$ is plausibly the ultimate cause of their remarkable SEDs and emission-line properties (see Section 1). Given the low X-ray fluxes $\left(\approx 10^{-15} \mathrm{erg} \mathrm{cm}^{-2} \mathrm{~s}^{-1}\right.$ from 0.5 to $\left.2.0 \mathrm{keV}\right)$ of the radioquiet PHL 1811 analogs, they will be excellent targets for future missions, e.g., the International $X$-ray Observatory (e.g., White et al. 2010), which will have far superior X-ray spectroscopic capability. Long-term X-ray monitoring of our PHL 1811 analogs will test whether they have state transitions like PHL 1092, and will help reveal whether instabilities of X-ray emitting ADCs universally exist in PHL 1811 analogs.

Finally, comparisons of high-quality spectra of PHL 1811 analogs and WLQs will help pin down any connection between them. UV spectroscopy of our PHL 1811 analogs covering the $\mathrm{O}$ vi line will reveal what fraction of them have very highly ionized UV absorption near the quasar redshift. Near-infrared spectroscopy of our PHL 1811 analogs covering the $\mathrm{H} \beta$ region should allow estimation of their $M_{\mathrm{BH}}$ and $L / L_{\mathrm{Edd}}$ values via the standard virial method (e.g., Vestergaard \& Peterson 2006; Shen et al. 2008), again testing for high $L / L_{\text {Edd }}{ }^{24}$ Such spectroscopy will also constrain their [O III] narrow-line regions, allowing

\footnotetext{
24 We note that application of the standard virial method for SMBH mass and $L / L_{\text {Edd }}$ determination will require caution, since extreme objects such as PHL 1811 analogs may not obey standard virial relations (cf. Section 6.4 of Richards et al. 2011). Comparison of $L / L_{\text {Edd }}$ values from the X-ray continuum based method and the virial method will thus be valuable as a consistency check.
} 
comparisons with eigenvector 1 of Boroson \& Green (1992). See the Appendix for near-infrared spectroscopy of J1521+5202. Near-infrared spectroscopy of additional high-redshift WLQs, covering the Fe II and Fe III transitions, will also reveal if the X-ray weak subset of this population is made up of PHL 1811 analogs (see Section 4.6).

We thank the anonymous referee for providing helpful comments. We thank A. C. Fabian, E. D. Feigelson, and B. P. Miller for helpful discussions. We acknowledge financial support from Chandra X-ray Center grant GO0-11010X (J.W., W.N.B.), NASA ADP grant NNX10AC99G (J.W., W.N.B.), and NSERC (P.B.H.).

Funding for the SDSS and SDSS-II has been provided by the Alfred P. Sloan Foundation, the Participating Institutions, the National Science Foundation, the U.S. Department of Energy, the National Aeronautics and Space Administration, the Japanese Monbukagakusho, the Max Planck Society, and the Higher Education Funding Council for England. The SDSS Web site is http://www.sdss.org/. The Hobby-Eberly Telescope (HET) is a joint project of the University of Texas at Austin, the Pennsylvania State University, Stanford University, Ludwig-Maximilians-Universität München, and Georg-AugustUniversität Göttingen. The HET is named in honor of its principal benefactors, William P. Hobby and Robert E. Eberly. The Apache Point Observatory $3.5 \mathrm{~m}$ telescope is owned and operated by the Astrophysical Research Consortium.

Note added in proof. $\mathrm{J} 1302+0030$ is one of the X-ray weak, high-redshift $(z=4.48)$ WLQs mentioned in Section 4.6. Shemmer et al. (2009) reported J1302+0030 to be X-ray weak by a factor of $\sim 10\left(\Delta \alpha_{\mathrm{ox}}=-0.38\right)$. Diamond-Stanic et al. (2009) found evidence for a strong blueshift $\left(\sim-5000 \mathrm{~km} \mathrm{~s}^{-1}\right)$ of the very weak ( $W_{r}=4.7 \AA$ ) C IV emission line in a Keck spectrum (their Figure 8 and Section 4.3). Our measurement of this spectrum gives a $C_{\text {IV }}$ blueshift of $-6150 \pm 850 \mathrm{~km} \mathrm{~s}^{-1}$ (68\% confidence level) and $W_{r}$ of $5.2 \pm 0.5 \AA$ (90\% confidence level). Therefore, J1302+0030 shows consistent behavior with PHL 1811 analogs in the $\Delta \alpha_{\text {ox }}-$ C IV blueshift $-W_{r}$ (C IV) parameter space, as shown in Figures 6 and 7. It can be considered to be a high-redshift PHL 1811 analog. The strong $\mathrm{C}$ IV blueshift and the X-ray weakness of J1302+0030 also support our unification scenario between WLQs and PHL 1811 analogs decribed in Section 4.6. We thank A. M. DiamondStanic for bringing this supporting evidence to our attention.

\section{APPENDIX}

\section{NEAR-INFRARED SPECTROSCOPY OF J1521+5202}

The X-ray properties of $\mathrm{J} 1521+5202$ were first reported by Just et al. (2007). This extremely optically luminous $\left(M_{i}=\right.$ $-30.19)$ quasar is exceptionally $\mathrm{X}$-ray weak by a factor of 34.5 (see Section 3). It has unusual UV emission-line properties (see Figure 2 and Table 3) like those of PHL 1811, for which the X-ray weakness may perhaps be attributed to a high $L / L_{\text {Edd }}$ value (Leighly et al. 2007b). To investigate the cause of the X-ray weakness for J1521+5202 and its relation to PHL 1811, we obtained its near-infrared spectrum ( $J H K$ bands) including coverage of $\mathrm{H} \alpha, \mathrm{H} \beta$, and [O III] $\lambda 5007$.

The near-infrared spectrum for $\mathrm{J} 1521+5202$ is shown in Figure A1(a) with a spectral coverage of $0.95-2.45 \mu \mathrm{m}$. We obtained this spectrum using the TripleSpec near-infrared spectrograph (Wilson et al. 2004) on the Apache Point Observatory (APO) 3.5 m telescope on 2009 May 19. We used a slit width
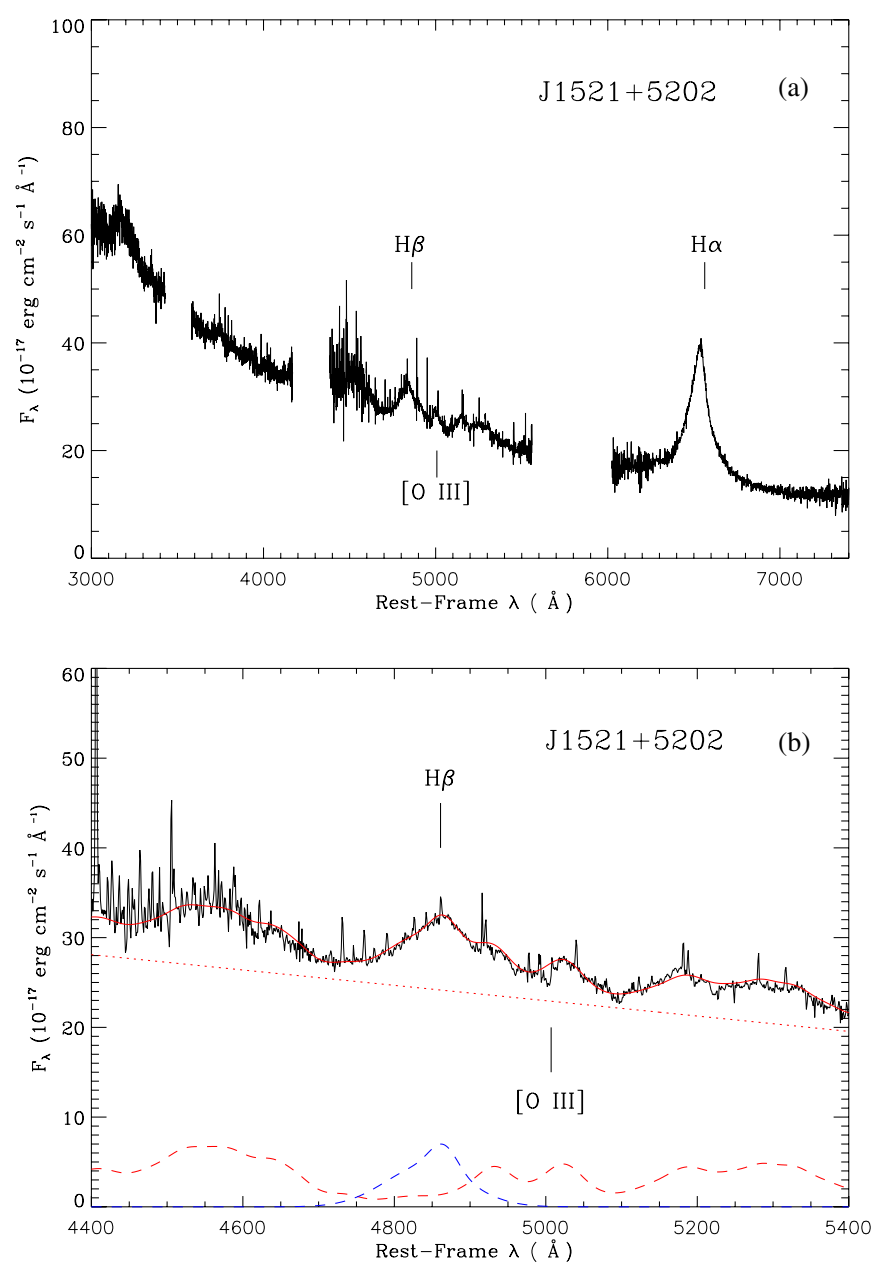

Figure A1. (a) Near-infrared spectrum of J1521+5202. The locations of the H $\alpha$, $\mathrm{H} \beta$, and [O $\mathrm{III}]$ emission lines are labeled. The gaps within the spectrum show the wavelength ranges that are affected most by the atmosphere. (b) Modeling of the spectrum between $4400 \AA$ and $5400 \AA$. The spectrum (black solid line) was resampled in bins of $1 \AA$ for clarity. The best-fit model (red solid line) consists of a continuum (red dotted line), a broadened Fe II emission complex (red dashed line), and an $\mathrm{H} \beta$ emission line (blue dashed line). The spectral resolution is $R \approx 2500$.

(A color version of this figure is available in the online journal.)

of $1^{\prime \prime} .7$, corresponding to a spectral resolution of $R \approx 2500$. This relatively broad slit width was chosen to maximize the signal-to-noise ratio in key spectral features such as the $\mathrm{H} \beta$ and [O III] $\lambda 5007$ emission lines. We observed J1521+5202 using an "ABBA" nodding pattern along the slit with each individual exposure lasting $200 \mathrm{~s}$. We collected twelve sets of observations in this way, giving $\approx 9600 \mathrm{~s}$ of total exposure for the $\mathrm{J} 1521+5202$ spectrum. Every $\approx 40 \mathrm{~min}$, we observed a calibration star (HD143817) for $40 \mathrm{~s}$. At the end of the observing run, we collected calibration flat frames. Sky lines in the images were used to perform wavelength calibration. We reduced the data following the standard procedures implemented in the 2009 May 1 version of the TripleSpecTool package. TripleSpecTool is a modified version of Spextool (Vacca et al. 2003; Cushing et al. 2004) that generates telluric-absorption corrected, absolute-calibrated spectra from TripleSpec images.

The near-infrared spectrum was modeled following the methods of Shemmer et al. (2004), as summarized below. Our model consists of (1) a linear continuum fitted between two narrow $( \pm 20 \AA)$ bands centered on $4700 \AA$ and $5100 \AA$ in the rest frame, 
(2) an Fe II emission template (Boroson \& Green 1992) broadened by convolution with a Gaussian profile with FWHM = $2700 \mathrm{~km} \mathrm{~s}^{-1}$, (3) the broad $\mathrm{H} \beta$ component represented by a Gaussian profile with $1200 \mathrm{~km} \mathrm{~s}^{-1} \leqslant \mathrm{FWHM} \leqslant 15000 \mathrm{~km} \mathrm{~s}^{-1}$, and (4) the narrow $\mathrm{H} \beta$ component and [O III] $\lambda \lambda 4959,5007$ represented by three Gaussian profiles with $300 \mathrm{~km} \mathrm{~s}^{-1} \leqslant$ FWHM $\leqslant 1200 \mathrm{~km} \mathrm{~s}^{-1}$. The three narrow Gaussian profiles were set to have the same width. The [O III] lines have the theoretical ratio $I([\mathrm{O}$ III $] \lambda 5007) / I([\mathrm{O}$ III $] \lambda 4959)=2.95$. The best-fit model is shown in Figure A1(b). The [O III] lines are undetected; we only obtained an upper limit of $W_{r}([\mathrm{O}$ III $] \lambda 5007)<1 \AA$. To set this upper limit, we assumed a Gaussian profile for [O III] $\lambda 5007$ with FWHM $=1000 \mathrm{~km} \mathrm{~s}^{-1}$, and we then determined the weakest such feature that would have been detected in our spectrum (e.g., Netzer et al. 2004).

We measured the systemic redshift using the best-fit $\mathrm{H} \beta$ line, $z=2.238$. This redshift is larger than that determined from the $\mathrm{Mg}$ II emission in the SDSS spectrum $\left(z_{q}=2.19\right)$. Using the $\mathrm{H} \beta$ redshift, the $\mathrm{C}$ IV line of $\mathrm{J} 1521+5202$ has an even larger blueshift $\left(-9400 \mathrm{~km} \mathrm{~s}^{-1}\right)$. The $\mathrm{C}$ IV blueshift remains strong $\left(-8400 \mathrm{~km} \mathrm{~s}^{-1}\right)$ using the redshift value estimated from $\mathrm{H} \alpha$. Like PHL 1811, J1521+5202 has very weak [O III] $\lambda 5007$ emission, and strong Fe II emission, which characterizes this source as an extreme eigenvector 1 object (Boroson \& Green 1992). The broad $\mathrm{H} \beta$ line of $\mathrm{J} 1521+5202$ has $\operatorname{FWHM}(\mathrm{H} \beta)=$ $5750 \mathrm{~km} \mathrm{~s}^{-1}$. We also measured the rest-frame equivalent width of $\mathrm{H} \beta, W_{r}(\mathrm{H} \beta)=30.7 \AA$, which is similar to those of the two WLQs in Shemmer et al. (2010), and smaller than that of PHL 1811 (50 ̊). Like WLQs, J1521+5202 has weaker H $\beta$ emission than most quasars (Boroson \& Green 1992; Shemmer et al. 2010). The strength of optical Fe II emission was measured to be $W_{r}(\mathrm{Fe}$ II $)=50.3 \AA$ between $4434 \AA$ and $4684 \AA$, yielding an $\mathrm{Fe} \mathrm{II} / \mathrm{H} \beta$ ratio of 1.6, which is similar to those for PHL 1811 and the two WLQs in Shemmer et al. (2010), and larger than those for most typical quasars (e.g., Netzer et al. 2004). This is expected given the weak [O III] emission and the anti-correlation between $W_{r}(\mathrm{O}$ III) and the $\mathrm{Fe}$ II $/ \mathrm{H} \beta$ ratio (see Figure 9 of Netzer et al. 2004).

The virial $M_{\mathrm{BH}}$ and $L / L_{\mathrm{Edd}}$ for $\mathrm{J} 1521+5202$ have been estimated from its rest-frame optical properties, $\mathrm{FWHM}(\mathrm{H} \beta)$ and $f_{\lambda}(5100 \AA)\left(2.20 \times 10^{-16} \mathrm{erg} \mathrm{cm} \mathrm{cm}^{-2} \mathrm{~s}^{-1} \AA^{-1}\right)$, using the empirical relation between BELR size and luminosity in Kaspi et al. (2005) modified by Bentz et al. (2009), and shown in Equations (1) and (2) of Shemmer et al. (2010). The bolometric correction to $v L_{v}(5100 \AA), f(L)=5.5$, was calculated with Equation (21) of Marconi et al. (2004). The results for $\mathrm{J} 1521+5202$ are $M_{\mathrm{BH}}=6.2 \times 10^{9} M_{\odot}$ and $L / L_{\mathrm{Edd}}=$ 0.81 . The $L / L_{\text {Edd }}$ value for $\mathrm{J} 1521+5202$ is not extraordinary compared to the high-redshift $(2<z<3.5)$, luminous $\left(L \gtrsim 10^{46} \mathrm{erg} \mathrm{s}^{-1}\right.$ ) quasars in Shemmer et al. (2004), for which the $M_{\mathrm{BH}}$ and $L / L_{\mathrm{Edd}}$ are measured in a similar single-epoch spectroscopy approach. However, we recognize the considerable uncertainties of $M_{\mathrm{BH}}$ and $L / L_{\mathrm{Edd}}$ estimated with this approach (at least a factor of 2-3 and perhaps much larger). When $L / L_{\text {Edd }}$ is close to unity, radiation pressure becomes important, which may present difficulties for the virial motion assumption for the gas around the SMBH (e.g., Marconi et al. 2008, 2009; but also see Netzer \& Marziani 2010). Furthermore, the calibration of $M_{\mathrm{BH}}$ with $\mathrm{FWHM}(\mathrm{H} \beta)$ and $L_{v}(5100 \AA)$ is performed using reverberation-mapping data for low-luminosity, low-redshift $(z<0.3)$ AGNs. Applying this calibration to high-luminosity, high-redshift AGNs may produce further uncertainties (e.g., Kaspi et al. 2007). ${ }^{24}$

\section{REFERENCES}

Abazajian, K. N., et al. 2009, ApJS, 182, 543

Arnaud, K. A. 1996, in ASP Conf. Ser. 101, Astronomical Data Analysis Software and Systems V, ed. G. H. Jacoby \& J. Barnes (San Francisco, CA: ASP), 17

Avni, Y. 1976, ApJ, 210, 642

Avni, Y., \& Tananbaum, H. 1986, ApJ, 305, 83

Becker, R. H., White, R. L., \& Helfand, D. J. 1995, ApJ, 450, 559

Begelman, M. C. 1978, MNRAS, 184, 53

Bentz, M. C., Peterson, B. M., Netzer, H., Pogge, R. W., \& Vestergaard, M. 2009, ApJ, 697, 160

Bergeron, J., \& Kunth, D. 1980, A\&A, 85, L11

Boroson, T. A., \& Green, R. F. 1992, ApJS, 80, 109

Bouchet, P., Lequeux, J., Maurice, E., Prevot, L., \& Prevot-Burnichon, M. L. 1985, A\&A, 149, 330

Brandt, W. N., \& Alexander, D. M. 2010, Proc. Natl Acad. Sci., 107, 7184

Brandt, W. N., \& Hasinger, G. 2005, ARA\&A, 43, 827

Brandt, W. N., Laor, A., \& Wills, B. J. 2000, ApJ, 528, 637

Chartas, G., et al. 2009, New Astron. Rev., 53, 128

Condon, J. J., Cotton, W. D., Greisen, E. W., Yin, Q. F., Perley, R. A., Taylor, G. B., \& Broderick, J. J. 1998, AJ, 115, 1693

Cushing, M. C., Vacca, W. D., \& Rayner, J. T. 2004, PASP, 116, 362

Diamond-Stanic, A. M., et al. 2009, ApJ, 699, 782

Dickey, J. M., \& Lockman, F. J. 1990, ARA\&A, 28, 215

Donato, D., Sambruna, R. M., \& Gliozzi, M. 2005, A\&A, 433, 1163

Eddington, A. S. 1922, MNRAS, 82, 432

Efron, B. 1979, Ann. Stat., 7, 1

Eisenstein, D. J., et al. 2011, AJ, submitted (arXiv:1101.1529)

Fabian, A. C., Iwasawa, K., Reynolds, C. S., \& Young, A. J. 2000, PASP, 112 1145

Feigelson, E. D., \& Nelson, P. I. 1985, ApJ, 293, 192

Frank, S., Mathur, S., Pieri, M., \& York, D. G. 2010, AJ, 140, 817

Freeman, P. E., Kashyap, V., Rosner, R., \& Lamb, D. Q. 2002, ApJS, 138, 185

Galeev, A. A., Rosner, R., \& Vaiana, G. S. 1979, ApJ, 229, 318

Gallagher, S. C., Brandt, W. N., Chartas, G., \& Garmire, G. P. 2002, ApJ, 567, 37

Gallagher, S. C., Brandt, W. N., Chartas, G., Priddey, R., Garmire, G. P., \& Sambruna, R. M. 2006, ApJ, 644, 709

Gallagher, S. C., Richards, G. T., Hall, P. B., Brandt, W. N., Schneider, D. P., \& Vanden Berk, D. E. 2005, AJ, 129, 567

Gallo, L. C., Grupe, D., Schartel, N., Komossa, S., Miniutti, G., Fabian, A. C., \& Santos-Lleo, M. 2011, MNRAS, 412, 161

Ganguly, R., \& Brotherton, M. S. 2008, ApJ, 672, 102

Garmire, G. P., Bautz, M. W., Ford, P. G., Nousek, J. A., \& Ricker, G. R., Jr. 2003, Proc. SPIE, 4851, 28

Gaskell, C. M. 1982, ApJ, 263, 79

Gehrels, N. 1986, ApJ, 303, 336

Gibson, R. R., Brandt, W. N., Gallagher, S. C., \& Schneider, D. P. 2009a, ApJ, 696, 924

Gibson, R. R., Brandt, W. N., \& Schneider, D. P. 2008a, ApJ, 685, 773

Gibson, R. R., Brandt, W. N., Schneider, D. P., \& Gallagher, S. C. 2008b, ApJ, 675,985

Gibson, R. R., et al. 2009b, ApJ, 692, 758

Hewett, P. C., \& Wild, V. 2010, MNRAS, 405, 2302

Hill, G. J., Nicklas, H. E., MacQueen, P. J., Tejada, C., Cobos Duenas, F. J., \& Mitsch, W. 1998, Proc. SPIE, 3355, 375

Hryniewicz, K., Czerny, B., Nikołajuk, M., \& Kuraszkiewicz, J. 2010, MNRAS, 404, 2028

Just, D. W., Brandt, W. N., Shemmer, O., Steffen, A. T., Schneider, D. P., Chartas, G., \& Garmire, G. P. 2007, ApJ, 665, 1004

Kaspi, S., Brandt, W. N., Maoz, D., Netzer, H., Schneider, D. P., \& Shemmer, O. 2007, ApJ, 659, 997

Kaspi, S., Maoz, D., Netzer, H., Peterson, B. M., Vestergaard, M., \& Jannuzi, B. T. 2005, ApJ, 629, 61

Komatsu, E., et al. 2009, ApJS, 180, 330

Kraft, R. P., Burrows, D. N., \& Nousek, J. A. 1991, ApJ, 374, 344

Krolik, J. H. 2007, in Black Holes: Spring Symposium at the Space Telescope Science Institute, ed. M. Livio (Cambridge: Cambridge Univ. Press), in press (arXiv:0709.1489)

Laor, A. 2000, New Astron. Rev., 44, 503

Latta, R. B. 1981, J. Am. Stat. Assoc., 76, 713

Lavalley, M., Isobe, T., \& Feigelson, E. 1992, in ASP Conf. Ser. 25, Astronomical Data Analysis Software and Systems I, ed. D. M. Worrall, C. Biemesderfer, \& J. Barnes (San Francisco, CA: ASP), 245

Leighly, K. M. 2004, ApJ, 611, 125 
Leighly, K. M., Halpern, J. P., Jenkins, E. B., \& Casebeer, D. 2007a, ApJS, 173, 1

Leighly, K. M., Halpern, J. P., Jenkins, E. B., Grupe, D., Choi, J., \& Prescott, K. B. 2007b, ApJ, 663, 103

Lyons, L. 1991, Data Analysis for Physical Science Students (Cambridge: Cambridge Univ. Press)

Maiolino, R., Salvati, M., Marconi, A., \& Antonucci, R. R. J. 2001, A\&A, 375 , 25

Marconi, A., Axon, D. J., Maiolino, R., Nagao, T., Pastorini, G., Pietrini, P., Robinson, A., \& Torricelli, G. 2008, ApJ, 678, 693

Marconi, A., Axon, D. J., Maiolino, R., Nagao, T., Pietrini, P., Risaliti, G., Robinson, A., \& Torricelli, G. 2009, ApJ, 698, L103

Marconi, A., Risaliti, G., Gilli, R., Hunt, L. K., Maiolino, R., \& Salvati, M. 2004, MNRAS, 351, 169

Miller, B. P., Brandt, W. N., Schneider, D. P., Gibson, R. R., Steffen, A. T., \& Wu, J. 2011, ApJ, 726, 20

Miller, K. A., \& Stone, J. M. 2000, ApJ, 534, 398

Miniutti, G., \& Fabian, A. C. 2004, MNRAS, 349, 1435

Miniutti, G., Fabian, A. C., Brandt, W. N., Gallo, L. C., \& Boller, T. 2009, MNRAS, 396, L85

Misawa, T., Eracleous, M., Chartas, G., \& Charlton, J. C. 2008, ApJ, 677, 863

Murray, N., Chiang, J., Grossman, S. A., \& Voit, G. M. 1995, ApJ, 451, 498

Mushotzky, R. 2004, in Supermassive Black Holes in the Distant Universe, ed. A. J. Barger (Astrophysics and Space Science Library, Vol. 308; Dordrecht: Kluwer), 53

Natali, F., Giallongo, E., Cristiani, S., \& La Franca, F. 1998, AJ, 115, 397

Nestor, D., Hamann, F., \& Rodriguez Hidalgo, P. 2008, MNRAS, 386, 2055

Netzer, H., \& Marziani, P. 2010, ApJ, 724, 318

Netzer, H., Shemmer, O., Maiolino, R., Oliva, E., Croom, S., Corbett, E., \& di Fabrizio, L. 2004, ApJ, 614, 558

Page, K. L., Reeves, J. N., O’Brien, P. T., \& Turner, M. J. L. 2005, MNRAS, 364, 195

Page, M. J., Carrera, F. J., \& Stevens, J. A. 2010, in AIP Conf. Proc. 1248, Xray Astronomy 2009: Present Status, Multi-wavelength Approach and Future Perspectives, ed. A. Comastri, L. Angelini, \& M. Cappi (Melville, NY: AIP), 391

Plotkin, R. M., Anderson, S. F., Brandt, W. N., Diamond-Stanic, A. M., Fan, X., MacLeod, C. L., Schneider, D. P., \& Shemmer, O. 2010a, ApJ, 721, 562 Plotkin, R. M., et al. 2010b, AJ, 139, 390

Ramsey, L. W., et al. 1998, Proc. SPIE, 3352, 34

Richards, G. T., et al. 2002, AJ, 123, 2945

Richards, G. T., et al. 2006, AJ, 131, 2766

Richards, G. T., et al. 2011, AJ, 141, 167

Risaliti, G., Salvati, M., \& Marconi, A. 2011, MNRAS, 411, 2223

Schlegel, D. J., et al. 2009, arXiv:0904.0468

Schmidt, M., \& Green, R. F. 1983, ApJ, 269, 352
Schneider, D. P., et al. 2001, AJ, 121, 1232

Schneider, D. P., et al. 2010, AJ, 139, 2360

Scott, J. E., Kriss, G. A., Brotherton, M., Green, R. F., Hutchings, J., Shull, J. M., \& Zheng, W. 2004, ApJ, 615, 135

Shemmer, O., Brandt, W. N., Anderson, S. F., Diamond-Stanic, A. M., Fan, X., Richards, G. T., Schneider, D. P., \& Strauss, M. A. 2009, ApJ, 696, 580

Shemmer, O., Brandt, W. N., Netzer, H., Maiolino, R., \& Kaspi, S. 2008, ApJ, 682,81

Shemmer, O., Netzer, H., Maiolino, R., Oliva, E., Croom, S., Corbett, E., \& di Fabrizio, L. 2004, ApJ, 614, 547

Shemmer, O., et al. 2006, ApJ, 644, 86

Shemmer, O., et al. 2010, ApJ, 722, L152

Shen, Y., Greene, J. E., Strauss, M. A., Richards, G. T., \& Schneider, D. P. 2008, ApJ, 680, 169

Shen, Y., et al. 2011, ApJS, 194, 45

Sobolewska, M. A., Gierliński, M., \& Siemiginowska, A. 2009, MNRAS, 394, 1640

Stark, A. A., Gammie, C. F., Wilson, R. W., Bally, J., Linke, R. A., Heiles, C., \& Hurwitz, M. 1992, ApJS, 79, 77

Steffen, A. T., Strateva, I., Brandt, W. N., Alexander, D. M., Koekemoer, A. M., Lehmer, B. D., Schneider, D. P., \& Vignali, C. 2006, AJ, 131, 2826

Stein, W. A., O’Dell, S. L., \& Strittmatter, P. A. 1976, ARA\&A, 14, 173

Stoughton, C., et al. 2002, AJ, 123, 485

Strateva, I. V., Brandt, W. N., Schneider, D. P., Vanden Berk, D. G., \& Vignali, C. 2005, AJ, 130,387

Su, D. Q., Cui, X., Wang, Y., \& Yao, Z. 1998, Proc. SPIE, 3352, 76

Telfer, R. C., Kriss, G. A., Zheng, W., Davidsen, A. F., \& Green, R. F. 1998, ApJ, 509,132

Tripp, T. M., Sembach, K. R., Bowen, D. V., Savage, B. D., Jenkins, E. B., Lehner, N., \& Richter, P. 2008, ApJS, 177, 39

Trump, J. R., et al. 2006, ApJS, 165, 1

Vacca, W. D., Cushing, M. C., \& Rayner, J. T. 2003, PASP, 115, 389

Vanden Berk, D. E., et al. 2001, AJ, 122, 549

Vestergaard, M., \& Peterson, B. M. 2006, ApJ, 641, 689

White, N. E., Parmar, A., Kunieda, H., Nandra, K., Ohashi, T., \& Bookbinder, J. 2010, in AIP Conf. Proc. 1248, X-ray Astronomy 2009: Present Status, Multi-wavelength Approach and Future Perspectives, ed. A. Comastri, L. Angelini, \& M. Cappi (Melville, NY: AIP), 561

Wilkes, B. J., \& Elvis, M. 1987, ApJ, 323, 243

Wilson, J. C., et al. 2004, Proc. SPIE, 5492, 1295

Worrall, D. M., Tananbaum, H., Giommi, P., \& Zamorani, G. 1987, ApJ, 313, 596

Wu, J., Brandt, W. N., Comins, M. L., Gibson, R. R., Shemmer, O., Garmire, G. P., \& Schneider, D. P. 2010a, ApJ, 724, 762

Wu, X.-B., et al. 2010b, Res. Astron. Astrophys., 10, 745

Yip, C. W., et al. 2004, AJ, 128, 2603

York, D. G., et al. 2000, AJ, 120, 1579 